\title{
YARARA: Significant improvement in RV precision through post-processing of spectral time series
}

\author{
M. Cretignier®, X. Dumusque ${ }^{\circledR}$, N. C. Hara ${ }^{\circledR}$, and F. Pepe ${ }^{\circledR}$
}

\begin{abstract}
Astronomy Department of the University of Geneva, 51 ch. des Maillettes, 1290 Versoix, Switzerland e-mail: michael.cretignier@unige.ch
\end{abstract}

Received 2 April 2021 / Accepted 14 June 2021

\begin{abstract}
Aims. Even the most precise radial-velocity instruments gather high-resolution spectra that present systematic errors that a data reduction pipeline cannot identify and correct for efficiently by simply analysing a set of calibrations and a single science frame. In this paper we aim at improving the radial-velocity precision of HARPS measurements by 'cleaning' individual extracted spectra using the wealth of information contained in spectral time series.

Methods. We developed YARARA, a post-processing pipeline designed to clean high-resolution spectra of instrumental systematics and atmospheric contamination. Spectra are corrected for: tellurics, interference patterns, detector stitching, ghosts, and fibre B contaminations, as well as more advanced spectral line-by-line corrections. YARARA uses principal component analysis on spectral time series with prior information to disentangle contaminations from real Doppler shifts. We applied YARARA to three systems, HD 10700, HD 215152, and HD 10180, and compared our results to the standard HARPS data reduction software and the SERVAL post-processing pipeline.

Results. We ran YARARA on the radial-velocity dataset of three stars intensively observed with HARPS: HD 10700, HD 215152, and HD 10180. For HD 10700, we show that YARARA enables us to obtain radial-velocity measurements that present an rms smaller than $1 \mathrm{~m} \mathrm{~s}^{-1}$ over the 13 years of the HARPS observations, which is $20 \%$ and $10 \%$ better than the HARPS data reduction software and the SERVAL post-processing pipeline, respectively. We also injected simulated planets into the data of HD 10700 and demonstrated that YARARA does not alter pure Doppler-shifted signals. For HD215152, we demonstrated that the 1-year signal visible in the periodogram becomes marginal after processing with YARARA and that the signals of the known planets become more significant. Finally, for HD 10180, the six known exoplanets are well recovered, although different orbital parameters and planetary masses are provided by the new reduced spectra.

Conclusions. The post-processing correction of spectra using spectral time series allows the radial-velocity precision of HARPS data to be significantly improved and demonstrates that for the extremely quiet star HD 10700 a radial-velocity rms better than $1 \mathrm{~m} \mathrm{~s}^{-1}$ can be reached over the 13 years of HARPS observations. Since the processing proposed in this paper does not absorb planetary signals, its application to intensively followed systems is promising and will certainly result in advances in the detections of the lightest exoplanets.
\end{abstract}

Key words. methods: data analysis - techniques: radial velocities - techniques: spectroscopic

\section{Introduction}

During the last 25 years, discoveries of exoplanets by radial velocity (RV) have considerably improved our knowledge about the diversity of exoplanetary systems. Nevertheless, detections of exoplanets are somewhat biased towards the detections of massive and short-periods planets. The discovery of a sub-metreper-second planetary signal with a period comparable to or longer than a year remains extremely challenging due to several factors, such as instrumental systematics, atmospheric contamination, and stellar activity.

Obtaining extremely precise radial velocities (EPRVs) is an art that requires: (1) a high-resolution super-stable spectrograph with high spectral fidelity to provide the cleanest possible spectra at the pre-calibration stage, (2) a data reduction pipeline that extracts, at best, the spectra from raw frames, corrects the instrumental systematics using a set of calibration frames, and extracts relevant information, such as the final RV of the spectrum, and (3) a post-processing of the RV time series to correct for stellar activity signals.

Due to tremendous progress in instrumentation, point (1) is becoming less of a concern due to the new generation of EPRV spectrographs, such as ESPRESSO (Pepe et al. 2014, 2021), EXPRES (Fischer et al. 2017), and MAROON-X (Seifahrt et al. 2018; Peck 2020; Sanchez et al. 2021), which have already demonstrated RV measurements with root mean square (rms) significantly smaller than $1 \mathrm{~m} \mathrm{~s}^{-1}$. The correction of stellar activity in the RV time series, point (3), has also been widely studied (e.g., Haywood et al. 2014; Grunblatt et al. 2015; Davis et al. 2017; Jones et al. 2017; Dumusque 2018; Cretignier et al. 2020a; Kosiarek \& Crossfield 2020; Collier Cameron et al. 2021). Only point (2), corresponding to a better extraction of the RV content of a spectra, has not been intensively studied in the last decade. Only a few papers have recently addressed this question (Dumusque 2018; Errmann et al. 2020; Zechmeister et al. 2020; Trifonov et al. 2020). However, this step is essential for better understanding stellar activity if an improvement in RV precision can be obtained.

It is known that different systematics on the spectra introduce RV effects. As examples, we can cite the colour variation in a spectrum with airmass or a change in atmospheric conditions, tellurics, or micro-tellurics (Artigau et al. 2014), as well as more instrument-related systematics specific to the HARPS instrument, such as the detector stitching (Dumusque et al. 2015). 
All these contaminations can be somewhat tricky to resolve when considering individual spectra, and corrections can only be achieved by successfully modelling the effects using prior information.

A methodology for correcting for these features is to consider spectral time series instead of individual spectra, as the former contain a richness of information far more extended than the latter. For example, this methodology shows its potential in the WOBBLE code (Bedell et al. 2019), which is designed to remove telluric lines by performing a principal component analysis (PCA), or in the Gaussian process (GP) framework developed by Rajpaul et al. (2020).

Following the WOBBLE approach, we developed a postprocessing RV pipeline called YARARA to cope with any known systematics in HARPS spectra. We show that by using the power of PCA on HARPS spectral time series, we can correct for any known systematics without the need for models for the different effects and with a limited amount of prior information. Such a data-driven approach is well suited to be applied to high signalto-noise ratio $(\mathrm{S} / \mathrm{N})$ spectra of ultra-stable spectrographs and to stars that have been intensively observed. Fortunately, such datasets are the only ones in which we can hope to search for sub-metre-per-second planetary signals, and thus the methodology used by YARARA is perfectly fitted to reach the best RV precision and look for the lightest exoplanets.

In this paper we apply our new post-processing pipeline YARARA to HARPS (Pepe et al. 2002a; Mayor et al. 2003; Pepe et al. 2003) data to demonstrate that further improvements can still be accomplished on HARPS RV measurements. The theory behind YARARA is described in Sect. 2. The different corrections performed in this work are described in the same order as they are implemented in the pipeline for HARPS, namely by correcting for: cosmic rays (Sect. 2.2), interference patterns (Sect. 2.3), telluric lines (Sect. 2.4), stellar lines variations (Sect. 2.5), ghosts (Sect. 2.6), stitchings (Sect. 2.7), simultaneous calibration contamination (Sect. 2.8), and continuum correction (Sect. 2.9). The line-by-line (LBL) RV derivation is described in 2.10, and a more advanced LBL correction is presented in Sect. 2.11. The applications of YARARA to three stellar systems are presented in Sect. 3 (Sect. 3.1 for HD 10700, Sect. 3.2 for HD 215152, and Sect. 3.3 for HD 10180). We then conclude in Sect. 4.

\section{Description of the pipeline}

The pipeline can be described as a sequential cascade of recipes applied to correct for the different systematics. The sequence described below is optimised for HARPS and was chosen based on two criteria: (i) the constrain we can have on the correction and (ii) the amplitude of the systematic in flux. Having a correction that is well constrained allows us to reduce the degeneracy with other recipes. Moreover, since we mainly use multi-linear regression to perform our corrections, it is necessary to correct first for the contaminations with the largest flux variance.

We note that different instruments might have a different sequential cascade depending on if the same or other contaminations exist and if they have different intensities.

The assumption behind the sequential approach is to assume that the different contaminations are orthogonal to each other. We will see further that this is the case if prior information (e.g., systematic structure, wavelength domain) is used to constrain the different recipes and if the baseline of the observations is long enough to avoid spurious correlations. As a consequence,
YARARA is mainly applicable to system intensively observed and for which a high $S / N(>100)$ can be obtained.

Our approach also assumes that the point spread function (PSF) does not vary significantly over time, and thus the data must come from the same stable spectrograph. We note that on HARPS, the change of the optical fibres in 2015 (Lo Curto et al. 2015) significantly changed the instrumental PSF. We therefore have to consider two instruments, one before and one after the upgrade. However, those two instruments are suffering from the same systematics, hence the same cascade of recipes can be applied. The only restriction is that spectral time-series objects before and after the fibre upgrade cannot be mixed in the pipeline and must be reduced separately.

In this work we call the map representing, in each row, a spectrum residual with respect to some reference a 'river diagram map'. We note that here we always represent river diagrams as the difference with respect to the median spectrum of the spectral time series. Since all the spectra are on a common wavelength grid, the spectral time series can be seen as a matrix. We refer to the variation in the flux $f\left(\lambda_{j}, t\right)$ as a function of the time $t$ for a specific wavelength $\lambda_{j}$ in this matrix as the 'wavelength column'. Each column is assumed independent for simplicity. The general mathematical framework of the corrections applied in YARARA take the shape of a multi-linear model fitted on the wavelength columns:

$\delta f\left(\lambda_{j}, t\right)=$ const. $+\sum_{i=1}^{N} \alpha_{i}\left(\lambda_{j}\right) \cdot P_{i}(t)$,

where $\alpha_{i}\left(\lambda_{j}\right)$ are the strength coefficients, fitted for using a weighted least-square considering as weight the inverse of the flux uncertainty squared, $P_{i}(t)$ are the elements of the basis fitted and $\lambda_{j}$ are the wavelengths either in the stellar or terrestrial rest frame. The data $\delta f\left(\lambda_{j}, t\right)$ can be either a spectra difference or a ratio with respect to a master spectrum $f_{0}\left(\lambda_{j}\right)$, depending on the systematics we want to correct for. Each time an RV shift of the spectra is performed, a cubic interpolation is used to resample the shifted spectra on a common $\lambda_{j}$ wavelength grid. The $P_{i}(t)$ basis will be made either of cross-correlation function (CCF) moments (contrast and FWHM) obtained by a weighted leastsquare Gaussian fit, or principal components of a weighted PCA (Bailey 2012) trained on a specific set of wavelengths $\lambda_{j}$. In all cases, the weights are defined as the inverse of the flux uncertainty squared.

\subsection{Data pre-processing}

To be efficient, spectral time-series objects must be continuum normalised, which is difficult due to the varying extinction of the Earth's atmosphere. A solution for echelle-grating instruments, consists in correcting the colour by scaling the spectra with a reference template or reference colour curve (e.g., Berdiñas et al. 2016; Malavolta et al. 2017). However, such corrections are usually performed order-by-order, which does not account for intra-order variations. A new approach based on alpha-shape regression was presented by Xu et al. (2019) on EXPRES spectra, where the authors demonstrated the better performance of alpha-shape algorithms compared to a more naive approach, such as iterative fitting methods (Tody 1986, 1993). Similarly, we developed a Python code called RASSINE (Cretignier et al. 2020b) and demonstrated its precision in flux on HARPS merged $1 \mathrm{D}$ spectra down to $0.10 \%$. RASSINE therefore appears as a well-suited tool for normalising spectra at the precision required to correct for the instrumental systematics. 


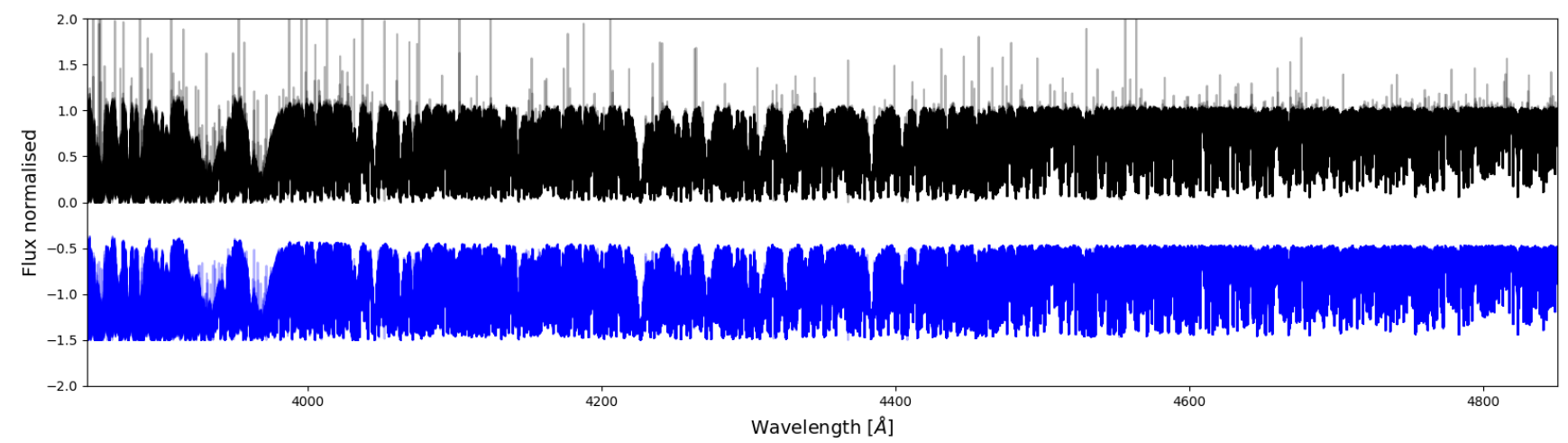

Fig. 1. First flux correction performed by YARARA to remove cosmic rays contamination. The 277 RASSINE-normalised spectra of HD 215152 are represented superposed before (black) and after (blue) YARARA cosmic ray cleaning. Cosmic rays are corrected by replacing every 5-sigma outlier larger than one by its value in the median spectrum.

The initial products given as input to YARARA are normalised merged 1D spectra. In this work, we started from HARPS merged 1D spectra produced by the official data reduction software (DRS), pre-processed them as follow, and then continuum normalised them using RASSINE. All the spectra are shifted in the stellar rest frame, night-drift corrected and evaluated on the same wavelength grid by a linear interpolation. Wavelengths longer than $6835 \AA$ were discarded due to the presence of the strong $\beta$ oxygen band. If the data contains large Doppler shift variations, typically larger than $\sim 5 \mathrm{~m} \mathrm{~s}^{-1}$ on HARPS, induced by binary companion or massive planets, the spectra are shifted to cancel this RV shift before being interpolated on the common wavelength grid. Smaller RV variations do not need to be corrected for since smaller RV amplitudes do not produce strong flux signatures in river diagram. Such a correction was unnecessary for the three stellar systems studied in Sect. 3. Spectra are then nightly stacked to improve the $\mathrm{S} / \mathrm{N}$ and finally normalised in automatic mode by RASSINE using the spectral time-series option, which allows the same local maxima to be selected on all the individual spectra (see Cretignier et al. 2020 b for more information).

We note that we decided to bin the spectra within a day, to help the normalisation process, as the upper envelope approach used by RASSINE to find the continuum is biased at low $\mathrm{S} / \mathrm{N}$. In addition, nightly binning helps in mitigating granulations and stellar oscillations (Bouchy \& Carrier 2001; Vázquez Ramió et al. 2005; Nordlund et al. 2009; Rieutord et al. 2010; Dumusque et al. 2011; Cegla et al. 2019), which induce RV variations with timescales smaller than a day.

\subsection{Cosmic ray correction}

Cosmic rays are high-energy particles that produce straight bright lines on raw images. When the anomaly created by a cosmic falls on the same pixel as the stellar spectrum, the real stellar flux is lost and the extracted stellar spectrum is significantly affected. This will thus strongly affect the derivation of RV locally on the spectra, as we will see further below ${ }^{1}$. In addition, in the context of YARARA, cosmic rays are seen as strong outliers and their impact has to be corrected for to improve the convergence of the different regressions that YARARA performs in the hereafter recipes.

\footnotetext{
1 We note that when deriving RVs using the CCF technique (Baranne et al. 1996; Pepe et al. 2002b), the perturbations induced by cosmic rays are generally averaged out.
}

In Cretignier et al. (2020b), the authors demonstrated that RASSINE is insensitive to cosmic rays since they can be flagged as local maxima with excessive derivative. Thus, cosmic rays will appear as spikes with values higher than one in normalised flux units (see Fig. 1). To detect them, for each wavelength column, we performed a 3-sigma clipping on the flux of all the spectra $^{2}$ and only removed the outliers larger than one. Such a technique removes efficiently the highest contaminations as visible in Fig. 1. We note that more outliers could be removed by pushing down the threshold on the sigma clipping; however, this could also remove contaminations other than cosmic rays, and removing them at this stage would make it more difficult to correct for them afterwards.

\subsection{Interference pattern correction}

On HARPS, an interference pattern was produced by mistake on FLAT calibration frames due to the use of a black body minus filter to balance the spectral energy distribution of a new Tungsten lamp installed in 2007. This filter was positioned in a collimated beam path, which produced the observed pattern following the thin-film interference law. This issue was solved during an upgrade of the instrument in August 2009, by moving the filter in a diverging beam. Hence, these systematics are only visible during two years of the HARPS observations (see Fig. 2). If the pattern frequency is low enough, RASSINE will naturally correct the oscillation as shown for ESPRESSO spectra in Allart et al. (2020). If the pattern has a high frequency, like for HARPS, with a periodicity shorter than one Angström, it needs to be corrected for.

The interference pattern has a unique frequency with time; however, the phase of the signal can change from day-to-day. We therefore decided to correct for the perturbing signal in the Fourier domain. To highlight at best the effect, we computed spectra ratio between each spectrum and the median of all excluding from the median all the spectra between 3 April 2007 and 24 July 2009, which are known to be affected. We then computed the fast fourier transform (FFT) of all the spectra ratio. For the spectra ratio that are showing excess of power around a width ${ }^{3}$ of $1.5 \mathrm{~mm}$ for a refractive index $n=1$ (see

\footnotetext{
2 We note that rather than using a standard deviation to perform the sigma clipping, we used 1.48 times the median absolute deviation in order to be less biased by outliers.

3 The interference periodicity can be found with the free spectral range law: width $=\frac{\lambda^{2}}{2 n \Delta \lambda}$.
} 

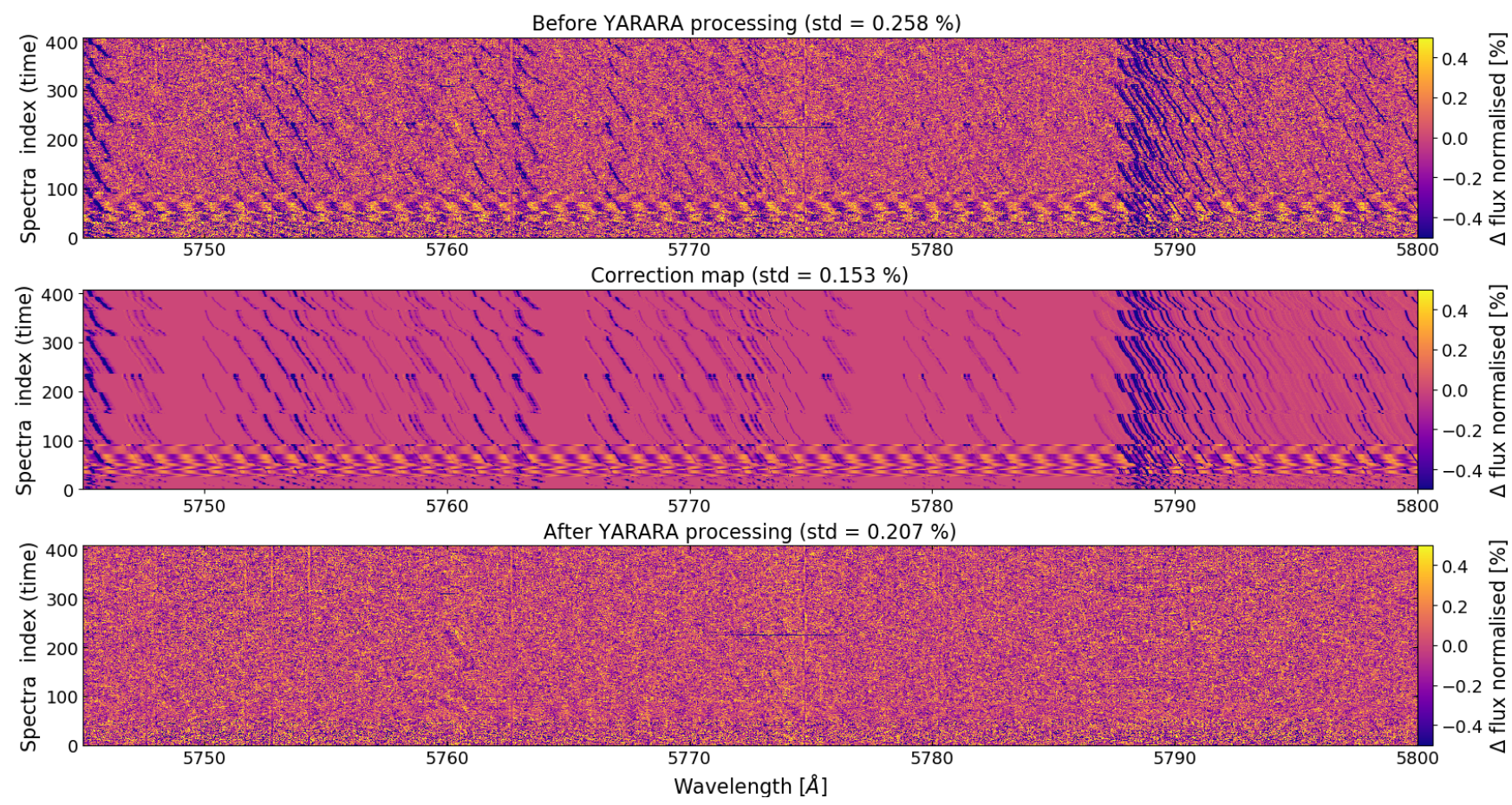

Fig. 2. Second and third flux corrections performed by YARARA to remove interference patterns and telluric absorption lines contaminations on HD 10700. The standard deviation of each residual map is indicated in the title. Top: river diagram before YARARA corrections. Spectra suffer from interference patterns on two observational seasons (from index 30 to 97). Middle: model fitted by YARARA for the contaminations. Bottom: residual river diagram after YARARA correction.

Fig. 9 in Cretignier et al. 2020b), we replaced the region with the excess of power by the same region seen in the FFT of the highest $\mathrm{S} / \mathrm{N}$ ratio spectra obtained outside of the contaminated time frame. The cleaned spectra are recovered by performing an inverse FFT followed by a multiplication with the median spectrum. This strategy successfully worked to remove the interference pattern as displayed in Fig. 2. We note that on HARPS we needed to treat the blue and red detectors differently as the gap in wavelength between the two charge-coupled devices (CCDs) was producing artefacts in the Fourier space.

\subsection{Telluric line correction}

Telluric lines are terrestrial atmospheric lines fixed in the barycentric Earth RV (BERV) rest frame that shift with respect to stellar lines and cross them due to the Earth reflex motion around the Sun, reducing the RV precision (Artigau et al. 2014; Cunha et al. 2014). In the visible, telluric lines come exclusively from water vapour or oxygen lines and both species will be corrected for differently. Indeed, whereas oxygen lines are stable with time and only show minor variations with airmass, water vapour lines exhibit large night-to-night and even intra-night variations (Li et al. 2018) depending on the humidity content in the air and the atmospheric conditions. Some methods based on line profile telluric modelling (Blake \& Shaw 2011; Bertaux et al. 2014; Smette et al. 2015) try to remove this contamination by using some prior information as those measured at the observational site (Baker et al. 2017). Unfortunately, such measurements are only providing local information on the ground, whereas the real information should be the column density of water along the direction of the observations, which is not a commonly derived quantity (Kerber et al. 2012). Another method consists in getting a high $\mathrm{S} / \mathrm{N}$ spectrum of an hot standard star in order to extract the telluric spectrum (e.g., Artigau et al. 2014), but such a method is time consuming and observationally expensive.

A more data-driven approach will take advantage that telluric lines are fixed in the BERV rest frame and can therefore be disentangled from the stellar spectrum. This method has been successfully used in Bedell et al. (2019), where the authors used a flexible data-driven model to remove the contamination. We use a similar approach, except that a PCA correction will solely be used as a second-stage correction, after applying a first step correction consisting of a multi-linear regression of the telluric CCF moments.

We use as prior information the position of the water and oxygen telluric lines as given by Molecfit (Smette et al. 2015). With these positions, a binary mask of water telluric lines is constructed in order to compute a telluric CCF. As a remark, the $\delta$ oxygen band around $5790 \AA$ is missing from the Molecfit database. We added the line positions by hand as they were clearly visible in our river diagram maps (see Fig. 2).

Because telluric lines present shallower depth than stellar lines, performing a CCF directly on the spectra will mainly contain stellar information. Switching to transmission spectra, dividing the observed spectra by a telluric-free stellar spectrum $f_{0}\left(\lambda_{j}\right)$ removes the contribution of stellar lines. Since the density of the telluric lines is quite low for the visible, we can build such a telluric-free stellar spectrum from the observations. This requires that for each specific wavelength column $\lambda_{j}$, at least one observation is not affected by tellurics. This hypothesis is equivalent to asking that the observations are probing a wide range of BERV values, where wide mean a range larger than the full width at half maximum (FWHM) of the telluric lines at the instrumental resolution. For HARPS, the FWHM is close to $3 \mathrm{~km} \mathrm{~s}^{-1}$ and therefore requires a minimum equivalent BERV span coverage. 
To perform our first level correction by CCF moments, a master stellar spectrum free of telluric lines is built by a masked median of the spectral time series, where a mask fixed in the BERV rest frame is applied to hide the telluric lines according to our database. The binary mask of water telluric lines is used to compute the CCF of the spectral time series divided by the master spectrum. All the lines of the mask have the same weight, meaning that the obtained CCF can be seen as an average telluric line. Since its equivalent width $(\mathrm{EW})$ can be interpreted as a proxy for water column density, this later is at first order proportional to the flux correction to apply. We note that a similar flux corrections is applied in Leet et al. (2019) with the SELENITE code, where the modelled correction is using as prior information both precipitable water vapour content and airmass.

To correct for telluric contamination, we shifted the ratio spectra in the BERV rest frame and performed a multi-linear regression (see Eq. (1)) by fitting both the contrast and FWHM time series of the telluric CCFs on each wavelength column. The correction was performed only if the Pearson coefficient between the flux column and the contrast or the FWHM was higher than 0.4. We note that telluric CCFs can still be contaminated by stellar lines and their moments can therefore be corrupted. This can fortunately be detected as a mismatch between the telluric CCF $\mathrm{RV}$ and the BERV. When those two quantities were diverging by more than $1 \mathrm{~km} \mathrm{~s}^{-1}$, we replaced the value of every moment by the median of the corresponding time series.

After performing this first correction, residuals were visible in telluric line cores, in particular at times where the telluric CCFs were really deep, which can be interpreted as a non-linear behaviour of water lines when the humidity content in the atmosphere is too high. We then decided to apply a second correction based on a weighted $\mathrm{PCA}^{4}$ (Bailey 2012). We performed a PCA on the previous residual matrix at the locations of clear telluric detection, which is estimated to be telluric deeper than $0.08 \%$ (see Appendix A). The PCA was weighted by the inverse of the flux uncertainty squared in order to prevent biasing the PCA algorithm with unusual low S/N observational epochs. The obtained PCA vectors were then fitted on each wavelength column of ratio spectra (see Eq. (1)). Only the three first principal components were fitted as adding more components did not reduce the variance further. The modelled telluric time series was extracted by taking the difference between the raw ratio spectral time series (in the terrestrial rest frame) and the residuals after CCF moments and PCA components fitted. The telluric timeseries model was then shifted back in the stellar rest frame and use to correct the ratio time series. The corrected spectra, in classical flux units, were recovered by multiplying the corrected ratio time series by the telluric-free spectrum $f_{0}\left(\lambda_{j}\right)$.

The spectral time series after this cleaning process can be seen in the lower panel of Fig. 2 and we observe that the adopted methodology successfully remove telluric contamination at the precision level of around $0.21 \%$. The second stage of correction can be seen as a refinement of the telluric correction and will mainly correct for residuals let by the first stage correction. This can be appreciated on the individual components extraction performed on HD 10700 (see Sect. 3.1) for which the RV amplitude of the second stage correction is one order of magnitude lower.

The same approach was used to correct for oxygen lines. On a final note, the method described here will certainly fail in the infrared where the lines density of water is too high. A pos-

\footnotetext{
4 The python code is freely accessible on GitHub: https://github. com/jakevdp/wpca
}

sible solution would be to perform a first order correction by telluric lines modelling (Seifahrt et al. 2010; Ulmer-Moll et al. 2019) before applying the PCA as a second-stage correction on the residuals.

\subsection{Point spread function variation and activity corrections}

The stellar CCF moments, such as the bisector inverse slope (BIS), the FWHM, the contrast, and the EW, have been widely used in the past to probe stellar activity (e.g., Queloz et al. 2001; Povich et al. 2001; Boisse et al. 2011). In particular, since those moments are known to contain no Doppler information, they are still frequently used nowadays, to highlight power excess or deficit at specific periods in periodograms, when the purpose is to assess the nature of periodic signals. The CCF can be seen as a weighted average stellar line and its variation therefore represents the average behaviour of stellar lines. In the context of stellar activity, several works have shown that photospheric lines were changing in morphology due to stellar activity (Stenflo \& Lindegren 1977; Cavallini et al. 1985; Basri et al. 1989; Brandt \& Solanki 1990; Berdyugina \& Usoskin 2003; Anderson et al. 2010; Thompson et al. 2017; Dumusque 2018; Wise et al. 2018; Ning et al. 2019; Löhner-Böttcher et al. 2019). In Cretignier et al. (2020a), we demonstrated how even symmetrical flux variations, which should a priori not induce an RV variation, can still introduce an RV effect if the line profile is asymmetric, such as in the case of a blended line (see also Reiners et al. 2013).

We note that in this paper we do not use the information of the CCF moments to correct for stellar activity; as for the quiet stars that will be presented in Sect. 3, no excess power is visible in any activity proxy, and thus stellar activity is not an issue. CCF moments that are tracing line-shape variations unrelated to Doppler shift (namely contrast and FWHM) can be used to probe and correct the variations in the instrumental PSF. To do so, we used an approach similar to the first-stage tellurics correction (see Sect. 2.4), except that the spectra are analysed in the stellar rest frame and spectra differences are used instead of spectra ratio as for tellurics. The stellar CCFs are extracted from the $\mathrm{G} 2$ or K5 CCF mask of HARPS depending on the stellar spectral type and a multi-linear regression (see Eq. (1)) of the contrast, the FWHM and the CaII S-index is fitted on each wavelength column.

The advantage of simultaneously fitting the S-index and moments of the CCFs is that whereas the former should only contain stellar activity effects, the latter can simultaneously contain instrumental and stellar activity components. Fitting all of them therefore leads to lifting the potential degeneracies that may exist. We can wonder if the fit in the flux of the S-index is motivated. Despite the higher sensitivity of the CaII H\&K lines to active regions, it is known that all photospheric lines change in depth with stellar activity (Basri et al. 1989). Even if photospheric and chromospheric variations can differ due to their different formation layers, several deep broad lines are also formed in the chromosphere as the CaII lines and will therefore undergoes similar variation with different strengths. This is confirmed in Fig. 3 where we represented the five years of observations of $\alpha$ Cen B and the correlations of each wavelength column with the $S$-index. Several wavelength columns show a significant correlation $(\mathcal{R}>0.4)$ with the $\mathrm{S}$-index. The modifications observed are equivalent to line core filling and line width broadening, which are effects already known from stellar activity on the 2010 dataset (Thompson et al. 2017; Wise et al. 2018; Ning et al. 2019; Cretignier et al. 2020a). We do not investigate 


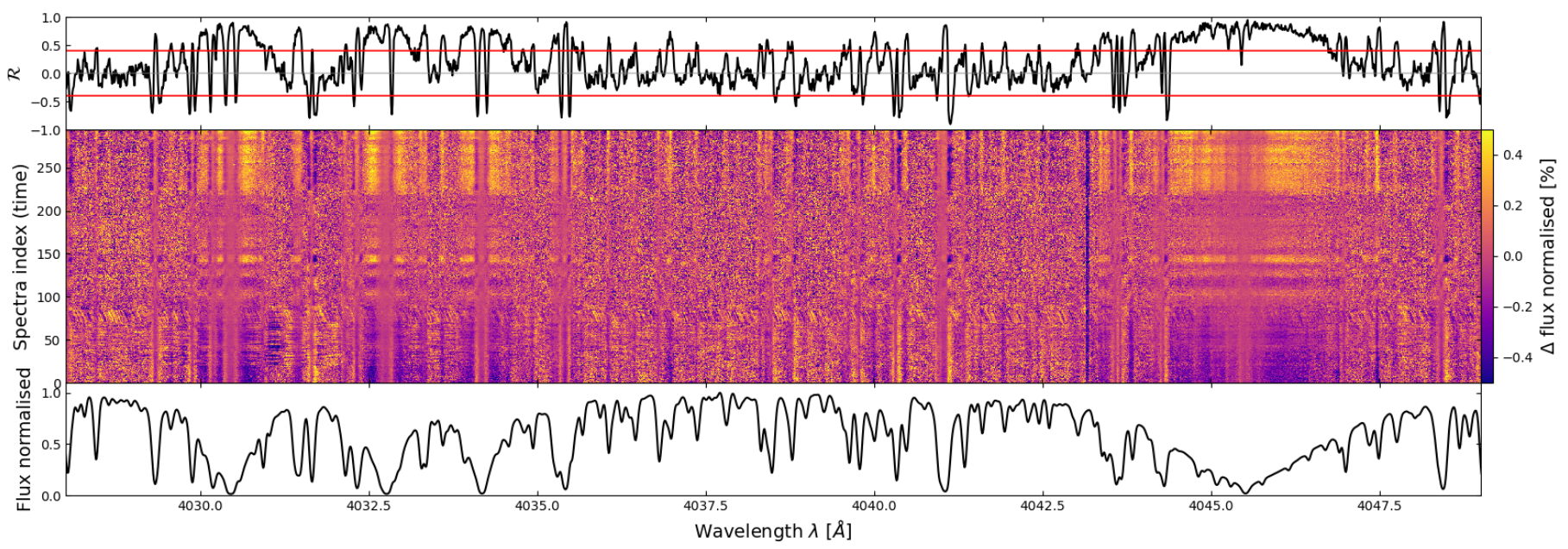

Fig. 3. Illustration of the flux variations induced by stellar activity on $\alpha$ Cen $\mathrm{B}$ and its correlation with the S-index on a small wavelength range in the extreme blue. Bottom: master normalised spectrum of $\alpha$ Cen B. Middle: river diagram of the five years of alpha Cen B observations. Some lines clearly show long trend flux variations corresponding to the magnetic cycle of the star. Top: Pearson correlation between the flux variations and the S-index showing, for several wavelengths, a significant correlation with $\left|\mathcal{R}_{\text {Pearson }}\right|>0.4$ (red horizontal lines). Such correlations were already reported by previous studies for the 2010 dataset, such as Thompson et al. (2017), Wise et al. (2018), Ning et al. (2019), and Cretignier et al. (2020a). The 2010 observational season is equivalent to index $102-150$ on the figure, for which the rotational modulation is clearly visible.
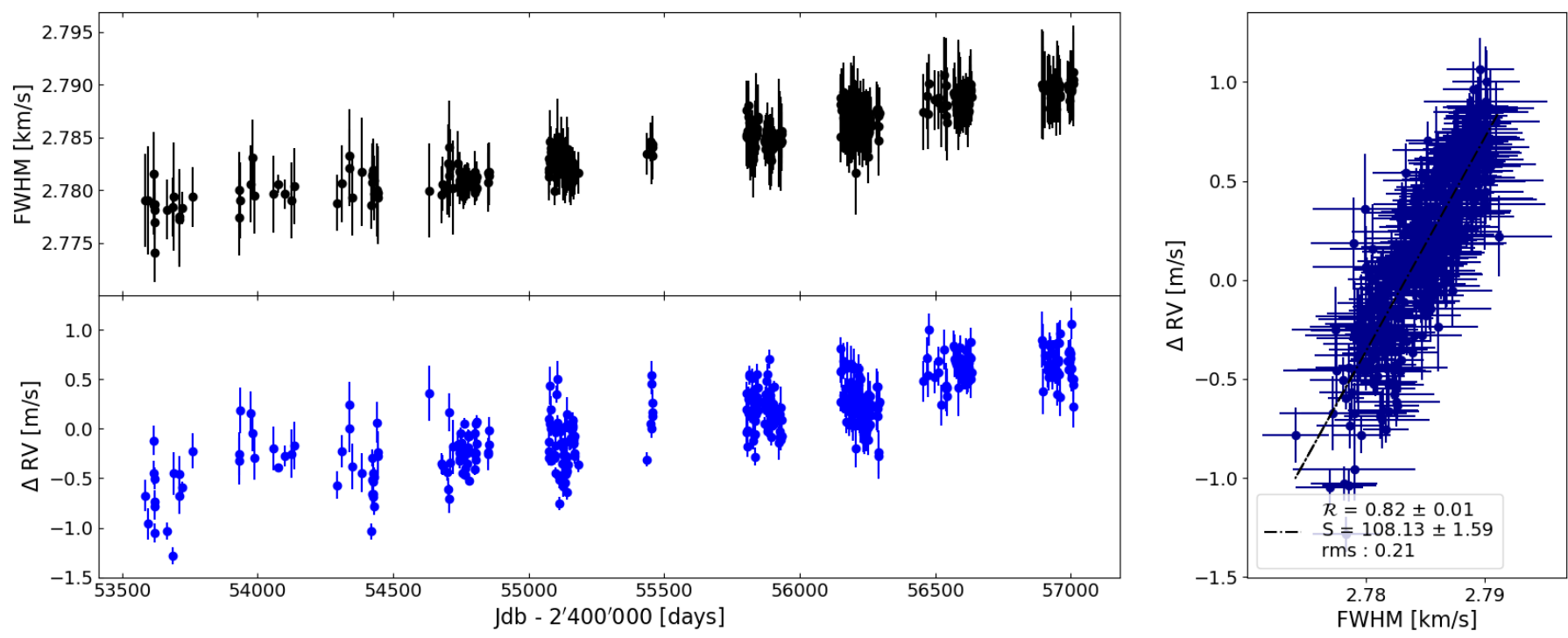

Fig. 4. Visualisation of the focus change due to HARPS ageing on the CCF FWHM and RV time series of HD 10700 obtained with the HARPS G2 mask. Top left: CCF FWHM time series of HD 10700. The star is known to be extremely quiet and the long-term trend observed is likely induced by the instrument ageing. The linear trend is about $1 \mathrm{~m} \mathrm{~s}^{-1}$ year $^{-1}$, which proves the impressive stability of HARPS (and HD 10700) over a decade. A similar though decreasing trend is observed in the CCF contrast. Bottom left: RV difference obtained on the RV before and after applying the YARARA correction to the spectral time series. YARARA allows a long-term linear drift of $10 \mathrm{~cm} \mathrm{~s}^{-1}$ year $^{-1}$ to be suppressed. Right: correlation plot of the previous time series, which shows the strong dependence between those two quantities, with a Pearson correlation coefficient of 0.82 . The $21 \mathrm{~cm} \mathrm{~s}^{-1}$ rms that we can see in the residuals can be explained by the simultaneous fit of the CCF contrast and CaII S-index along with the FWHM or bad fit on some wavelength columns.

further stellar activity since such an analysis is left for a future dedicated paper and the targets selected hereafter are quiet stars.

It is important to mention that CaII H\&K lines can be contaminated by ghosts (see Sect. 2.6), which are not yet corrected for at that moment. Hopefully for HARPS, as demonstrated in Appendix B, ghosts never fall simultaneously on the CaII H and CaII K lines. In addition, the contamination depends on the systemic $\mathrm{RV}\left(\mathrm{RV}_{\text {sys }}\right)$ of the observed star that will shift the spectra and thus the position of the cores of the CaII H\&K lines relative to ghosts that are fixed in the barycentric rest frame. Since both chromospheric lines react in a similar way, the activity proxy that we fit will be made of either one line (CaIIH or CaIIK) or both combined (S-index), depending on the $\mathrm{RV}_{\text {sys }}$ of each star (see Fig. B.1). For an instrument strongly contaminated by ghosts, a careful extraction of the $\mathrm{S}$-index should be performed (Dumusque et al. 2021).

When analysing the data of the extremely quiet star HD 10700, it is possible to observe the change of HARPS's focus as a function of time, which is associated with the ageing of the instrument. Indeed, the CCF FWHM (top left panel of Fig. 4) and the contrast show a net increase and decrease with time, respectively. This is coherent with a slight decrease in the 

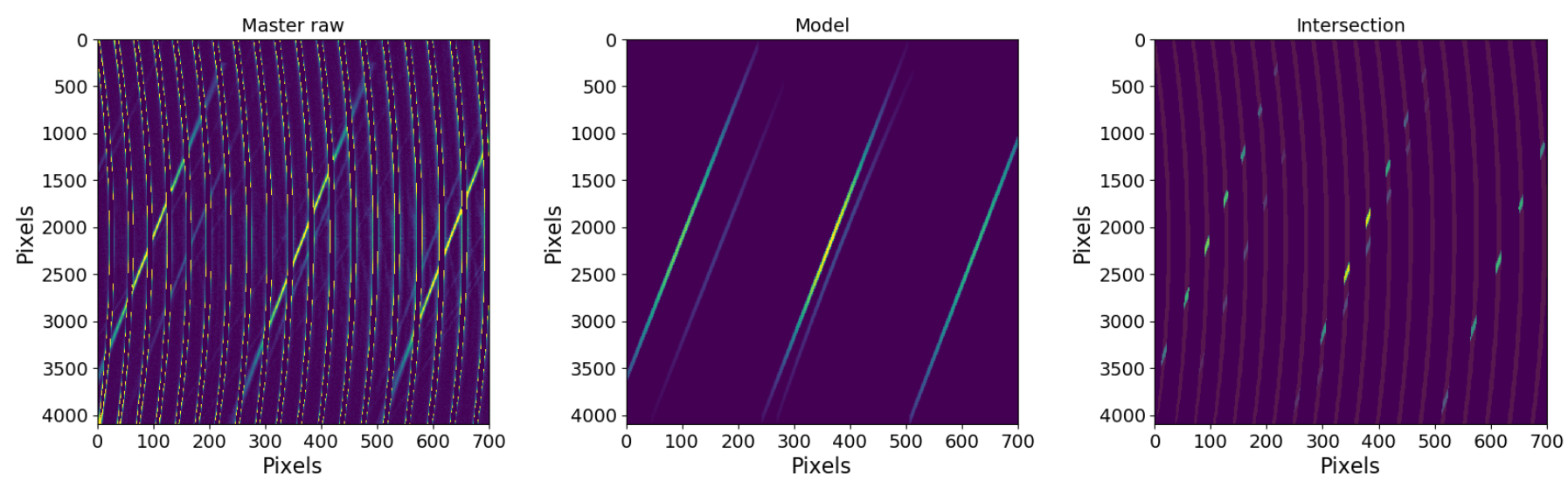

Fig. 5. Derivation of the static product used in the fourth flux correction performed by YARARA dedicated to ghosts contamination. Left: master raw image of the blue detector obtained after stacking two months of FLAT calibration frames when only the science fibre was illuminated. The echelle spectrograph orders are the vertical parabola, which are masked here to highlight the ghosts visible in the background. Middle: ghosts as fitted by our models. Right: map of ghost contamination indicating the location of the ghost of the science fibre on itself. Bright colour highlights the intersection of the ghosts with the physical orders. A similar map was derived for the ghosts of the simultaneous calibration fibre crossing the order of the science fibre.

HARPS resolution with time, equivalent to a PSF broadening. The EW, which is at first order the product of the FWHM and the contrast, does not show such a long-term trend. It is, however, relevant to note that the FWHM variation observed is only about $1 \mathrm{~m} \mathrm{~s}^{-1}$ per year. It confirms the remarkable stability of the instrument along its lifetime, as well as the incredible stability of the star itself.

To measure the RV impact of the instrument ageing, we computed the RV before and after applying the flux correction using the HARPS G2 cross-correlation mask and measured the difference between both RV time series. This difference is displayed in the bottom left panel of Fig. 4 and is strongly correlated with the FWHM variation as we can see in the right panel of the same figure, with a Pearson correlation coefficient of 0.81 . The RV impact of the instrument ageing is extremely small, with a linear slope of only $10 \mathrm{~cm} \mathrm{~s}^{-1}$ year $^{-1}$. We note that even if such a linear correlation between FWHM and RV can theoretically be used to correct for instrumental ageing on the RV time series, correcting for the effect at the flux level is a more robust way of handling this issue.

\subsection{Ghosts correction}

Ghosts are spurious reflections of the physical orders occurring inside the instrument, which produce tilted-duplicated images of the spectral orders on the CCD. Due to their reflection nature, ghosts are at maximum a hundred times less luminous than the original echelle orders, as measured on a master FLAT raw frame (see Fig. 5). As a consequence, ghosts are difficult to distinguish from the read-out-noise of the detector on individual calibration frames, and no calibration frames can be produced to highlight 'only' ghosts since those are secondary reflection of the main echelle orders. However, ghosts always appear at the same position on the detector and can thus be highlighted on high $\mathrm{S} / \mathrm{N}$ images obtained after stacking several raw frames together. As an example, we show on the left panel of Fig. 5 an image obtained after stacking two months of HARPS FLAT calibrations raw frames and we clearly see the contamination induced by ghosts as oblique bright lines.

The ghosts' contamination is not corrected in the HARPS DRS because it is difficult to deal with it at the extraction level due to the relative small angle between ghosts and the main echelle orders, but mainly because when observing a star, the ghosts will be a copy of the stellar spectrum itself, and not the 'flat' spectrum of a tungsten lamp used for flat-fielding. To our knowledge, no study on HARPS has analysed to which main echelle orders the ghosts belong, thus making any correction extremely difficult.

A proper way of correcting for ghost contamination in the context of RV measurements was never investigated further as ghosts only contaminate spectral regions with very low flux, such as the extreme blue in the stellar observation, and those regions do not contribute significantly to the extracted RVs. However, ghosts can significantly contaminate the cores of the CaII H\&K lines in the extreme blue, at 3834 and $3969 \AA$, which are used to derive the calcium activity $S$-index, the main stellar activity indicator for G-K dwarfs (Wilson \& Vainu Bappu 1957; Oranje 1983; Baliunas et al. 1988). This is the case in the HARPS-N solar observation, where a ghost correction technique was performed to derive a calcium activity index free of contamination (see Dumusque et al. 2021).

To be able to derive a calcium activity index free of systematics, but also to obtain meaningful LBL RV information in the extreme blue, we decided to correct for ghost contamination at the flux level with YARARA. The first step consists in modelling the ghosts on the raw frames (middle panel) in order to determine their intersection with the science orders (right panel). The modelling of ghosts is detailed in Appendix B. To obtain the wavelengths of a spectrum that are contaminated by ghosts, we took advantage of the HARPS instrumental stability, which allows us to use a static wavelength solution to map contamination from pixels to wavelengths. As the contamination is additive, we looked for the contamination in spectrum difference time series, obtained by subtracting to each spectrum a master built as the median of all spectra. The river diagram map of spectrum differences shifted in the BERV rest frame is displayed in the top panel of Fig. 6 . Around 3819 and $3849 \AA$, we see ghost contamination from the science fibre (fibre A), and around 3822 and $3851 \AA$, ghost contamination from the reference fibre (fibre B). We then selected, at the location of ghost crossings, all the wavelength that where showing a relative flux variation more important that $1 \%$ and trained a weighted PCA (Bailey 2012) to model the ghost contamination. Finally, we fitted for all the wavelengths columns located in a ghost crossing region, the first 

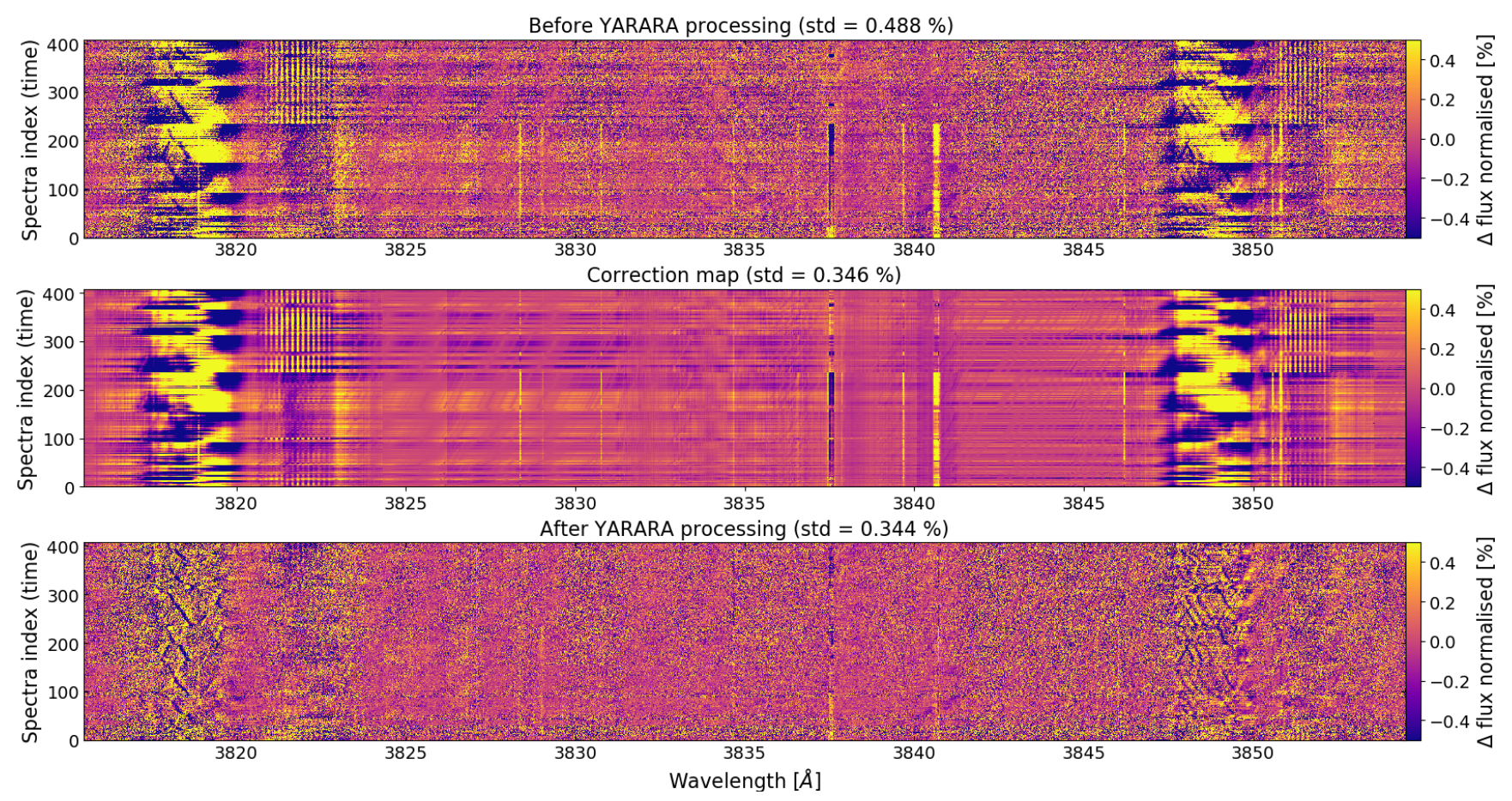

Fig. 6. Ghost, thorium-argon, and continuum corrections performed by YARARA on HD 10700. The river diagram is produced in the BERV rest frame. Top: spectral time series before YARARA corrections. Spectra suffer from thorium-argon contamination (bright vertical lines, for example close to $3840 \AA$ ) and Fabry-Pérot contamination by a ghost of the simultaneous calibration fibre (vertical bright comb around 3822 and $3851 \AA$ ). The strong contamination around 3819 and $3849 \AA$ As due to two ghosts of the science fibre. Middle: model of the contamination fitted by YARARA. Bottom: residual river diagram after YARARA correction.

three PCA components for ghosts of fibre A and the first two for ghosts of fibre B (see Eq. (1)).

We note that around 3819 and $3849 \AA$, the ghost contamination of fibre A presents an emission/absorption pattern, which is unexpected. Naively, we would only expect an emission, such as for thorium-argon contamination, but on a much larger region (see the top panel of Fig. 6 and Sect. 2.8). As this is not the case, one explanation is that the master spectrum subtracted is not free of ghosts. Indeed, since ghosts cross the orders with a small penetration angle $\left(\sim 5^{\circ}\right)$, their contamination is present over about one hundred pixels. In consequence, even if the star studied present large BERV values (at maximum $\sim 60 \mathrm{~km} \mathrm{~s}^{-1}$, thus 73 HARPS pixels, as a pixel is $\sim 820 \mathrm{~m} \mathrm{~s}^{-1}$ ), significant wavelength ranges are always contaminated by ghosts. An option would be to optimise the way the reference master is built; however, such a solution will not work for stars with a small span in BERV. It is important to note that this issue will provide an inaccurate correction though one that is still very precise, which is the important factor when deriving RVs. The same problem does not occur for ghost contamination of fibre B since this fibre is illuminated by either a thorium-argon lamp or a Fabry-Pérot etalon, both of which present distinct emission peaks and not a continuous emission, unlike the ghosts of fibre A, which are reflections of a stellar spectrum. This explains why we fitted the first three PCA components for fibre A, and only the first two for fibre B.

The principal component model of ghost contamination from fibre B clearly captures the Fabry-Pérot structure as visible by the emission comb around 3822 and $3851 \AA$ in the middle panel of Fig. 6. This structure is only visible once the Fabry-Pérot has been routinely used on HARPS, which happened in 2012. We can see a few remaining residuals in the lower panel of the same figure inside the ghost A regions, which appear as dark lines perfectly phase-folded at one sidereal year. Those lines are the stellar lines of the ghosts contaminating the science fibre. Since both the ghost stellar lines and the science stellar lines are shifted with the BERV, those lines are not fixed in the BERV rest frame and determining their rest frame is out of the scope of the present paper.

Although ghost contamination is strongly mitigated after YARARA correction, the structured residuals could still impact the RV of spectral lines appearing in the contaminated regions. We will see that those residuals impact the derived RV of spectral line in a characteristic way different from a Doppler shift, and we correct for those at a later stage (see Sect. 2.11).

\subsection{Stitching correction}

Stitching are detector systematics induced when manufacturing a CCD. The HARPS $4096 \times 4096$ CCD is a mosaic composed of eight blocks of $512 \times 1024$ pixels, which are not perfectly aligned together. The pixels located at the stitching of the different blocks have a different size than the pixels within blocks, due to manufacturing limitation. If not properly accounted for when extracting spectra and measuring a wavelength solution, this difference in size will induce an RV variation at a period of one year, as first shown by Dumusque et al. (2015). In this paper, the authors strongly mitigate the induced one-year signal by simply rejecting from the RV computation the part of the spectrum affected by the stitching effect. However, this rejects a significant amount of RV information, and a better way to handle this problem is to measure the size of the pixels at the different stitching location and include it in the wavelength solution derivation (Coffinet et al. 2019). We will see below that stitching effect can also be efficiently corrected for at the flux level. 

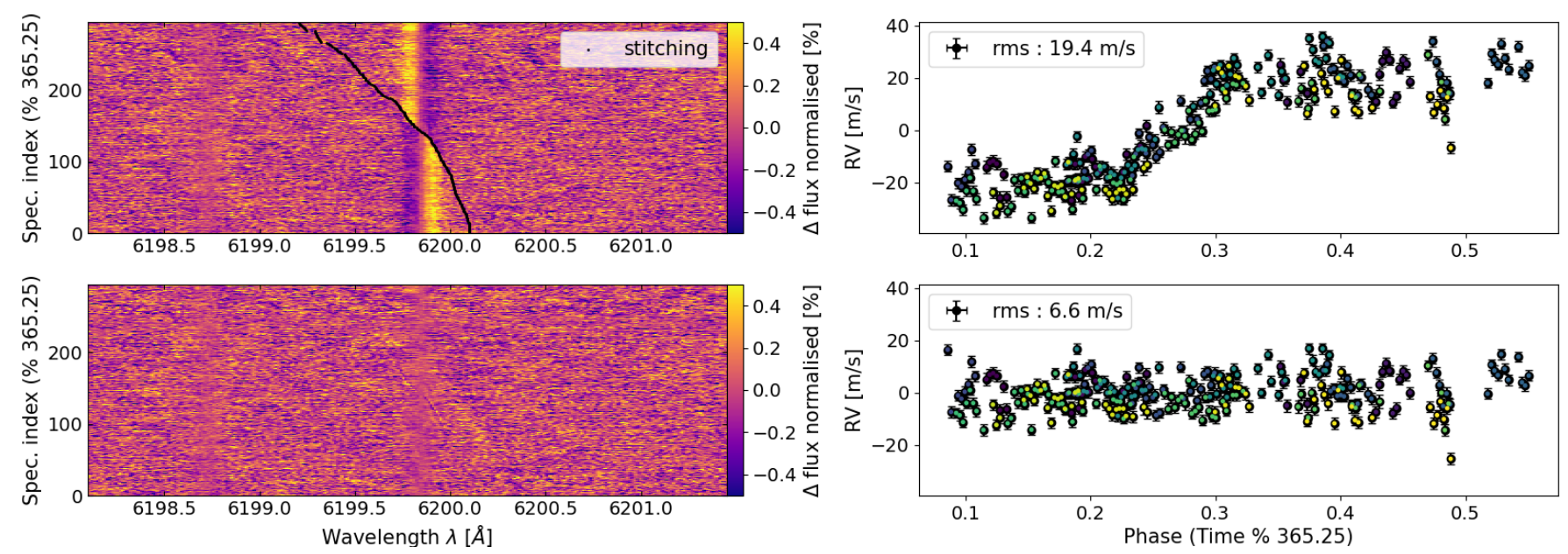

Fig. 7. Stitching correction performed by YARARA on the five years of HD 128621 data for the $6200 \AA$ stellar line described in Dumusque et al. (2015). The time dimension is phase-folded at 1 year. Top left: spectral time series before YARARA corrections. The position of the stitching is indicated by the black curve and is used to mark the delimitation of the step function used for correction (see text). Bottom left: same as top left but after YARARA processing. Top right: LBL RV of the line crossing the stitching. Colour encodes the unfolded time. Bottom right: same as top right but after YARARA processing. The RV rms has been reduced from $19.4 \mathrm{~m} \mathrm{~s}^{-1}$ down to $6.6 \mathrm{~m} \mathrm{~s}^{-1}$. The LBL morphological correction (see Sect. 2.11) mitigates the rms even further, down to $5.6 \mathrm{~m} \mathrm{~s}^{-1}$.

In Fig. 7, we display the same stitching-crossing stellar line at $6200 \AA$ for HD 128621 as reported in Dumusque et al. (2015). The left panel show the flux anomaly created by the wrong wavelength solution at the stitching position before and after YARARA correction. We note that the time dimension in those river diagram maps is phase-folded at one year. The right panel of the same figure show the LBL RV of the $6200 \AA$ spectral line, phase-folded at 1 year, before and after YARARA correction. It is clear that before correction the RV of the line shows a sigmoid-like shape variation, with a jump of $40 \mathrm{~m} \mathrm{~s}^{-1}$ between phases where the stitching is on both sides of the spectral line. From the left panel of Fig. 7, we can see that for each wavelength columns, the effect in flux is a step function, where the position of the step correspond to the stitching location. Instead of fitting for a step function that could be biased by outliers, we computed the median below and above the stitching boundary for each wavelength column crossing a stitching and subtracted this model. We clearly see that the stitching flux anomaly is strongly mitigated in the river diagram map residuals. When deriving the LBL RV of the $6200 \AA$ spectral line using the spectra corrected from YARARA, the original $19.4 \mathrm{~m} \mathrm{~s}^{-1} \mathrm{RV}$ rms of the line is strongly reduced down to $6.6 \mathrm{~m} \mathrm{~s}^{-1}$. As in the case of ghost contamination, the remaining small residuals can be corrected further when studying the LBL RVs as the induced effect will be different from a Doppler shift. Performing LBL morphological corrections (see Sect. 2.11) manages to correct the systematic down to an rms of $5.6 \mathrm{~m} \mathrm{~s}^{-1}$.

\subsection{Thorium-argon correction}

A thorium-argon lamp was used on HARPS (before the use of the Fabry-Pérot etalon Wildi et al. 2011) as a reference calibrator to measure the drift of the instrument throughout the night. To do so, the light from the thorium-argon lamp was injected in the reference fibre (fibre B) of the spectrograph, to simultaneously measure the instrumental drift along with stellar observations. During a stellar observation, the reference thorium-argon spectrum on fibre B presents, however, a significant number of saturated lines that 'bleed' on the detector and contaminate the nearby science fibre. Lowering the flux of the lamp to avoid saturation is not an option, unfortunately, as otherwise the $\mathrm{S} / \mathrm{N}$ of the Thorium spectrum would not be sufficient to reach the precision required to measure the tiny drifts of the spectrograph overnight.

The HARPS DRS corrects for this effect by using thoriumargon contamination calibrations. Those calibrations have only fibre B illuminated by the thorium-argon lamp. The contamination of the lamp at the position of the science fibre is extracted and then fitted for in stellar observations. Unfortunately, these calibration frames were only rarely produced on HARPS, which implies an imperfect correction as line intensities change with time due to the ageing of the lamp or due to a change of lamp, which happened a couple of times throughout HARPS's lifetime $^{5}$. We can clearly see at wavelengths $3837 \AA$ and $3841 \AA$ in Fig. 5 that the use of static contamination products do not provide an excellent correction.

To perform a better correction, we first used the available thorium-argon contamination calibrations to select the spectral regions contaminated by saturated lines. We used a 1.5 interquartile (IQ) sigma-clipping to detect anomalous flux intensities compared to the noise level. The IQ being more robust statistically to detect outliers (Upton \& Cook 1996). We then trained a weighted PCA on the selected wavelength columns in the BERV rest frame, and corrected for the contamination by fitting the first two PCA components on all the wavelength columns. We decided to fit the model on the full wavelength domain instead of on flagged regions, like for ghosts, since detecting the contamination in the calibrations frames is difficult. To prevent the first two PCA components and to fit for any type of systematics, we performed the correction only if the Pearson coefficient with one of either PCA vector was higher than 0.4.

\subsection{Continuum improvement and iterative processing}

So far, a recipe has always built its own master spectrum to be as free as possible from the contamination it was correcting for.

\footnotetext{
5 A history of lamp changes for HARPS can be found here: http://www.eso.org/sci/facilities/lasilla/instruments/ harps/inst/monitoring/thar_history.html
} 
Since spectra at the end of the pipeline are cleared at best of all the observed systematics, the final master spectrum is necessarily better that the one available at the beginning of the pipeline. For that reason, a second iteration trough the different recipes by using always the master spectrum obtained at the end of the first iteration is performed. As an example, the first correction that benefits from the new master spectrum is the Savitchy-Golay continuum fit performed in RASSINE. Indeed, since telluric lines are initially not corrected for, they induce a poor continuum normalisation around telluric bands.

After the second iteration, no significant flux variation is remaining in the river diagram maps, except small residuals at ghost crossing and a few outliers. To get rid of these remaining systematics, we finally perform a 2-sigma clipping on each wavelength columns and replace outliers by the median value of the corresponding wavelength column, as done for cosmic correction (see Sect. 2.2), with the exception that no condition on the flux level is imposed.

\subsection{Stellar mask optimisation and LBL RVs}

YARARA was developed to locally extract the RV information in a stellar spectrum, as done in Dumusque (2018). One of the final purposes of the pipeline is to produce better LBL RVs since spectra are precisely normalised thanks to RASSINE and spectra are cleared out of their main contaminations. A great advantage after YARARA post-processing is that the available master spectrum is expected to be almost completely free of all known contaminations. Such a master can thus be used to perform cross-correlation and derive an RV value per spectrum (Anglada-Escudé \& Butler 2012; Gao et al. 2016; Zechmeister et al. 2020), or derive LBL RVs.

LBL RV extraction by first order flux linearisation (Bouchy et al. 2001) only requires three quantities: (1) a master template used to measure the flux variation $\delta f$ between a spectrum and the master, (2) the line profile gradient $\partial f / \partial \lambda$ to account properly for the weighting of the RV information and (3) the window centred on a spectral line to extract its RV signals. A few surprising results can be found if a generic mask is used instead of a tailored line selection in LBL RV analysis. Indeed, due to blended lines that are in some way unique for each star depending on the stellar temperature, metallicity, $v \sin (i)$ or instrumental resolution, a generic mask will in some case produce spurious RV signals, which was demonstrated in Cretignier et al. (2020a). We note that similarly to Dumusque (2018) and Cretignier et al. (2020a), Lafarga et al. (2020) showed that tailored lines selections, produced by their public code RACCOON, can be useful to boost the S/N and decrease the photon noise, which produces in general better RV precision.

We implemented in YARARA a similar line window selection as described in Cretignier et al. (2020a). This consists simply in selecting for each line, the wavelength range between the two local maxima surrounding the local minimum formed by the line core defined as the local minimum. Doing so, blended lines will sometimes be grouped together but this is not an issue as soon as signals specific to each line profile do not try to be interpreted Cretignier et al. (2020a). We note that the obtained selection of lines does not exclude spectral regions strongly contaminated by telluric lines, as commonly performed by generic line masks, and therefore the line selection of YARARA can be used to extract RV information with the highest $\mathrm{S} / \mathrm{N}$.

If we want to use the obtained line selection as a mask for performing cross-correlation, we need to obtain the proper depth of the line relative to the continuum as this is used as weight when deriving the CCF (Pepe et al. 2002b). In addition, if we want to derive LBL RVs using this line selection, we also need to estimate at best the continuum and depth of the spectral lines as the LBL technique uses the line profile gradient $\partial f / \partial \lambda$ as weight. In regions where a continuum exists, the normalisation by RASSINE allows the proper estimate for the continuum and line depth to be derived. However, this is no longer the case in the blue part of solar-like spectra or in the spectra of $M$ dwarfs as no continuum exists due to the strong absorption. This problem is solved by measuring the deviation of the estimated RASSINE continuum with respect to a stellar template close to the effective temperature of the star, and correcting for it, as shown in (Cretignier et al. 2020b). As an example, we shown in Appendix C and Fig. C.1, the YARARA correction for continuum opacity in the stellar spectrum of an M1.5 dwarf.

\subsection{LBL morphological corrections}

All the previous corrections managed at some level to remove efficiently the different observed contaminations. In some cases, however, we noticed that some residual signals were still present. Those are due to (1) degeneracies between the recipes, which typically occurs when several contaminations are located at the same wavelength position, (2) more complex patterns, such as the ones created when ghosts contaminate stellar line (see Sect. 2.6), and (3) regions not flagged by our static products. Unlike a Doppler shift, those residuals will change the shape of spectral lines with time, which can be tracked and corrected for using a few metrics that describe line morphology.

We extracted three morphological parameters to measure a change in the line profile not related to a Doppler shift. We first separate the left and right wing of a spectral line with respect to its core defined as the local minimum of the line, and then measure the total flux on the left $(\mathrm{L})$ and right $(\mathrm{R})$ wings. We then build the $\mathrm{L}+\mathrm{R}$ and $\mathrm{L}-\mathrm{R}$ metrics to account for asymmetric variations. Our third parameter is the depth of the stellar line, fitted by a parabola on the line core with a window of $\pm 2 \mathrm{kms}$.

Although a Doppler shift does not affect the line depth, it does affect $L$ and $R$, and thus our metrics $L+R$ and $L-R$. To correct for Doppler shift variation, we built a calibration curve for each stellar line in our line selection. To do so, we shifted all the spectral line with values ranging from -100 to $100 \mathrm{~m} \mathrm{~s}^{-1}$ by step of $0.5 \mathrm{~m} \mathrm{~s}^{-1}$ and measured for each of them how $\mathrm{L}-\mathrm{R}$ and $\mathrm{L}+\mathrm{R}$ behaved. We then linearly interpolated each relation on a finest grid of $1 \mathrm{~cm} \mathrm{~s}^{-1}$. Once we have those calibration curves, the $\mathrm{L}+\mathrm{R}$ and $\mathrm{L}-\mathrm{R}$ metrics for a given spectral line can be obtained by (1) deriving its LBL RV, (2) read on its calibration curve the $\mathrm{L}-\mathrm{R}$ and $L+R$ values for this $R V$ and (3) measuring the difference between the measured and theoretical values. By doing this calibration and correction, we ensure that our morphological proxies $\mathrm{L}+\mathrm{R}$ and $\mathrm{L}-\mathrm{R}$ are planetary-free. We demonstrated in Sect. 3 that neither known planets, nor injected ones, disappeared at the end of our pipeline, which is a first validation of the method.

Those morphological parameters, phase-folded with a period of 1-year, are represented in Fig. 8 for the stellar line at $3849 \AA$ in HD 10700 HARPS observations. This line was clearly showing residuals after ghost correction in Fig. 5. By observing the LBL $\mathrm{RV}$, after flux correction (bottom centre panel), several 'mountain peaks' are clear visible, a signature coherent with several flux anomalies crossing the stellar line. Because this variation is not equivalent to a pure Doppler shift, the same pattern can be observed in the L-R proxy (top centre panel). By linearly decorrelating the LBL with the three morphological proxies, the RV 

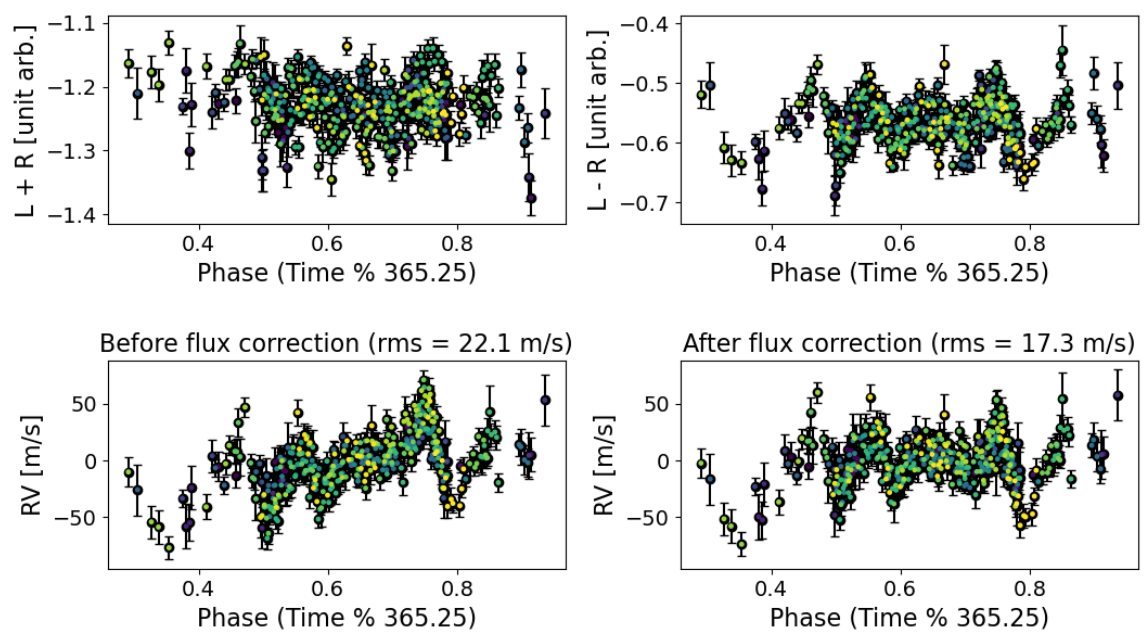
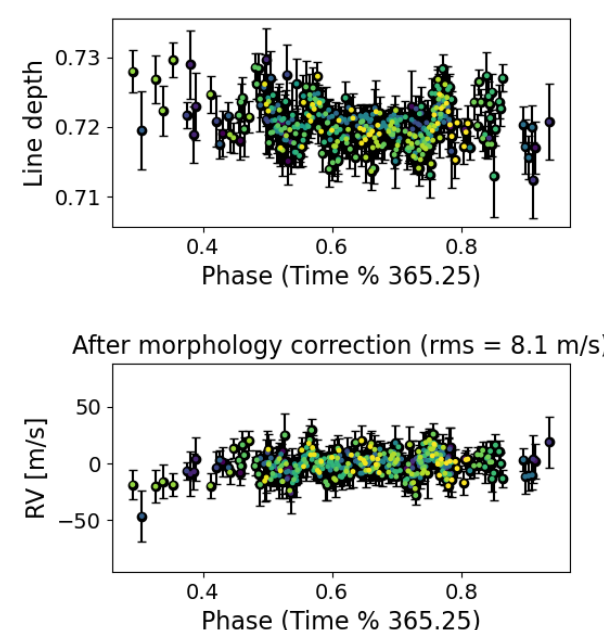

Fig. 8. Morphological corrections performed by YARARA on HARPS observations of HD 10700 for the $3849 \AA$ line contaminated by a ghost of the science fibre. The time dimension was phase-folded at one sidereal year. Top left: $\mathrm{L}+\mathrm{R}$ area deviation with respect to the Doppler shift calibration curve (see main text for the precise definition), which is therefore similar to its EW. Top middle: $\mathrm{L}-\mathrm{R}$ area deviation with respect to the Doppler shift calibration curve. Top right: line depth of the line computed by a parabola fitting on the line core. Bottom left: LBL RV of the line before any correction. Bottom middle: LBL RV after YARARA flux corrections. The stellar lines present in the ghost are still visible. Bottom right: LBL RV after multi-linear de-correlation with the three morphological proxies. The $8.1 \mathrm{~m} \mathrm{~s}^{-1} \mathrm{rms}$ of the RV residuals is compatible with the median of the RV uncertainties.

contamination still remaining after YARARA flux correction is highly reduced. We note that for this line, the RV rms is mitigated from 22.1 to $17.3 \mathrm{~m} \mathrm{~s}^{-1}$ after YARARA flux correction, and down to $8.1 \mathrm{~m} \mathrm{~s}^{-1}$ after LBL morphological correction.

The same morphological correction was systematically applied for all of the 3770 stellar lines in our line selection for HD 10700. We displayed in Fig. 9 how such a correction is helpful to correct for 1-year period contaminations. We fitted a 1-year sinusoid on each LBL RV and studied the correlation between the time series and the best-fitted model. In the different panels of this figure we show, for each line, the weighted Pearson correlation coefficient between the LBL RVs and the fitted sinusoid as a radial coordinate as well as the phase of the fitted sinusoid as a polar coordinate. The colour represents the semi-amplitude of the fitted sinusoid. We defined a line as significantly affected if its power exceeds a false alarm probability (FAP) level of $1 \%$ at 365.25 days. We see in Fig. 9 that before any correction is applied (top left panel), 1071 stellar lines exhibit a significant 1-year power, some of them with semi-amplitudes larger than $10 \mathrm{~m} \mathrm{~s}^{-1}$. The third quartile (Q3) of the amplitude distribution is $9.1 \mathrm{~m} \mathrm{~s}^{-1}$. Despite the large number of lines contaminated, it is important to highlight that since the phases between all the signals are not coherent, the final amplitude of the contamination is roughly of $1 \mathrm{~m} \mathrm{~s}^{-1}$. Those lines can be contaminated by any effect fixed in the BERV rest frame, in our case tellurics, stitchings, ghosts or thorium-argon contaminations. Those contaminations should be significantly mitigated after YARARA flux correction (top right panel), which is indeed the case as only 428 stellar lines still present a significant 1-year signal after flux correction, and Q3 is reduced down to $5.36 \mathrm{~m} \mathrm{~s}^{-1}$. After morphological LBL correction, the number of significantly affected lines drops to 227 and Q3 to $3.2 \mathrm{~m} \mathrm{~s}^{-1}$. Figure 9 therefore highlights that YARARA, through flux and line morphology corrections, is able to strongly mitigate instrumental systematics with a oneyear period.

The 227 spectral lines that are still affected are so far not understood. After investigations, no particular instrumental systematics are expected on the detector at their positions. Also, since their contamination is not corrected for by our morpho- logical correction, it indicates that the flux contamination is too low to be detected by our morphological proxies. It is further confirmed by the observation that such remaining 1-year lines are not found after flux corrections for HD 215152 for which the median $\mathrm{S} / \mathrm{N}$ of the spectral time series $(S / N \sim 120)$ is ten times lower than for HD $10700(S / N \sim 1000)$. We note that when fitting for ghosts localisation on the high $\mathrm{S} / \mathrm{N}$ master FLAT, some barely visible ghosts were excluded as considered as negligible (see Appendix B). Those could therefore be responsible for the remaining affected spectral lines.

To remove from our final selection part of those 227 contaminated lines, we proceed as followed. All the lines presenting a power at 1-year higher than a FAP level of $1 \%$ are first removed and the average RV signal $\mathrm{RV}_{m}$ of the complementary lines' selection is computed. This $\mathrm{RV}_{m}$ signal was then subtracted in each individual LBL RVs, in order to remove any real planetary signal if any, before redetermining the power at 1-year for each individual line. The motivation behind subtracting $\mathrm{RV}_{m}$ is to prevent absorbing any real planetary signal appearing at a period of 1 -year or corresponding harmonics (see the planetary injection simulation performed in Sect. 3.1). Lines with a 1-year power higher than 1\% FAP level are removed such that only 103 lines still present significant 1-year power for HD 10700 and Q3 is decreased down to $2.5 \mathrm{~m} \mathrm{~s}^{-1}$ (bottom right panel). This line rejection step is performed by the pipeline for all the stars, but only stars with exquisite $\mathrm{S} / \mathrm{N}$, such as HD 10700, will have some lines rejected, generally fewer than a hundred lines.

An explanation for that remaining 1-year signal is the inaccurate wavelength solution due to the sparse location of thoriumargon lines and their uncertainties on their absolute wavelength values. In Coffinet et al. (2019), the authors showed that echelleorder wavelength solution by polynomial fit were inaccurate by dozens $\mathrm{m} \mathrm{s}^{-1}$ compared to the wavelength solution obtained with a laser frequency comb. The inaccurate wavelength solution will at term introduce systematics at 1-year of small amplitudes on LBL RVs since the BERV will move the stellar lines on this inaccurate wavelength solution. Opposite to ghosts, all the lines will be in phase with the BERV. This seems to be observed on the bottom right panel of Fig. 9 by an elongation of the cloud along 
Input data
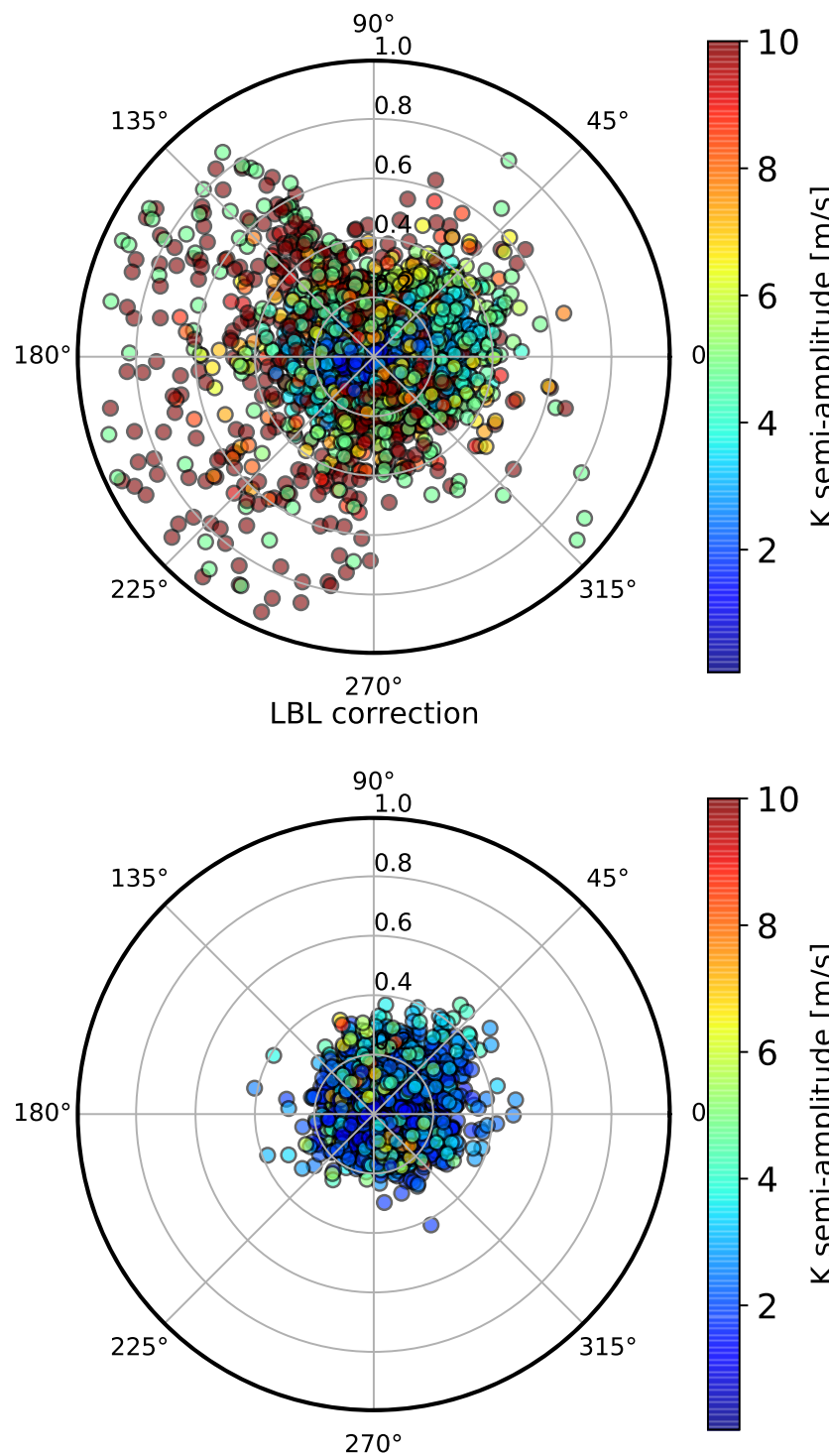

Flux correction
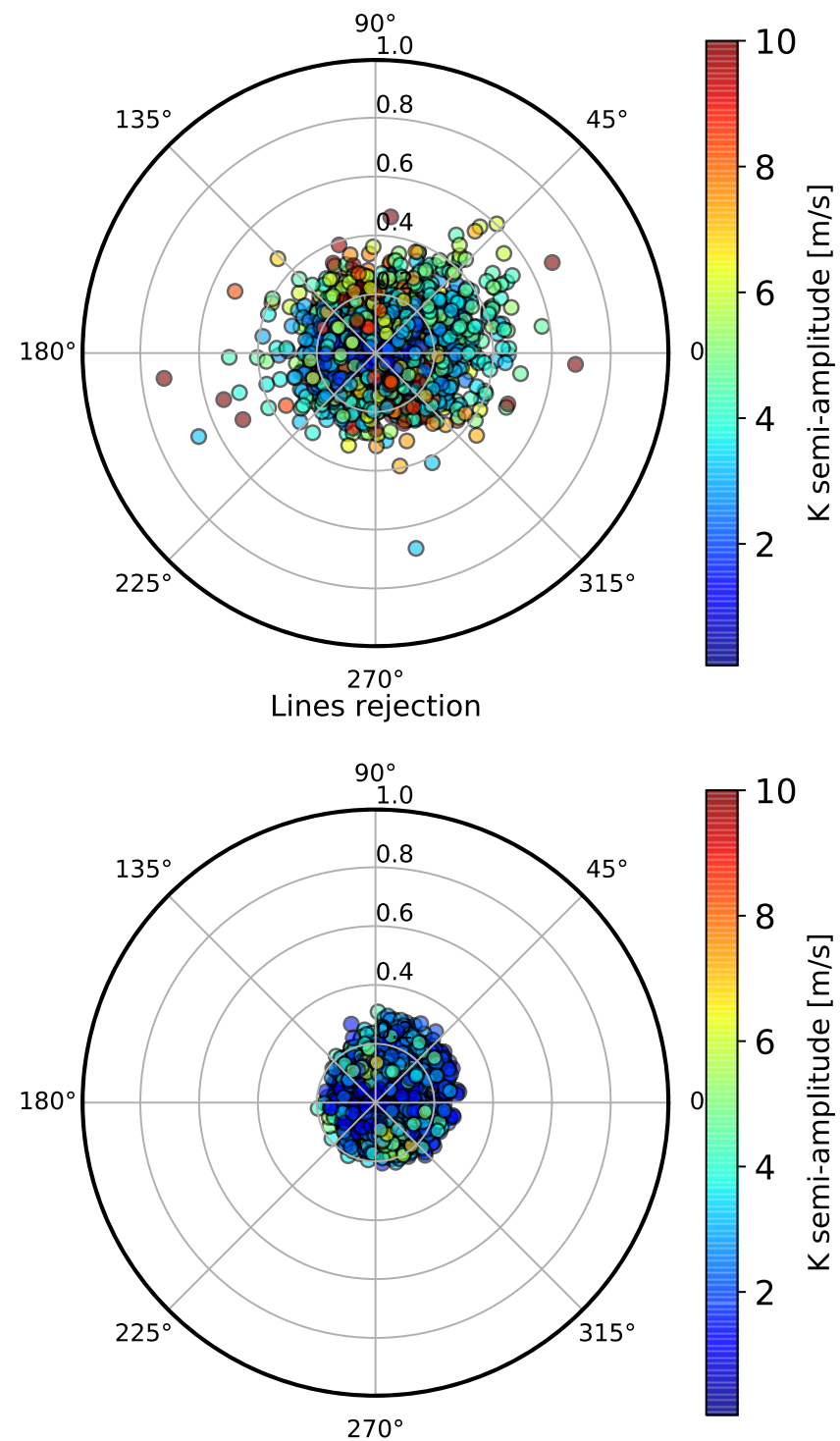

Fig. 9. Polar periodogram representation of LBL RV time series for HD 10700. The polar periodogram is focused at 1 year and represents the Pearson coefficient with a 1-year sinusoid as radial coordinates (see the main text) and the phase of the signal as the polar angle. Among the 3770 stellar lines, the numbers of lines presenting a Pearson coefficient higher than 0.4 are, respectively from top left to bottom right: 339 , 59, 15 and 0. Top left: input dataset without any YARARA correction. Top right: dataset after YARARA flux correction. Bottom left: dataset after the LBL morphological corrections. Bottom right: dataset after lines with a significant 1-year power are rejected. Most of the 1-year-affected lines have been cleaned out.

the $45^{\circ}$ direction, which is almost consistent with the BERV phase of $30^{\circ}$ that we can obtain by doing a similar analysis not shown here. Due to the small amplitudes of the signals expected $\left(<5 \mathrm{~m} \mathrm{~s}^{-1}\right.$ from the LBL RVs), it is rather hard to confirm this hypothesis.

More sophisticated LBL RV corrections could be developed from this point, but those will be described in a forthcoming paper dedicated to stellar activity correction. For now, this stage is representing the ending point of our pipeline. Its application to several HARPS targets is presented in the next section.

\section{Results}

In this section, we analysed the data of three stars intensively observed with HARPS, to test the performances of YARARA. We first looked at the data of HD 10700 in Sect. 3.1 as it appears to be the quietest star in the entire HARPS sample, with an RV rms close to $1 \mathrm{~m} \mathrm{~s}^{-1}$ over more than a decade. We compared the RVs obtained after YARARA processing, with the ones from the classical HARPS DRS and the SERVAL post-processing pipeline (Zechmeister et al. 2020; Trifonov et al. 2020). As this star does not show any significant planetary signal, we also injected a fake planetary signal to demonstrate that YARARA processing was not altering planetary signals. We then analysed HD 215152 in Sect. 3.2, to demonstrate that YARARA strongly mitigate the impact of yearly signals induced in this dataset mainly by detector stitchings. Finally, we studied HD 10180 in Sect. 3.3 that is known to harbour six planets. We show that all the planets are recovered with high confidence after YARARA processing, and the obtain orbital parameters are similar with the published values, however, with some interesting differences. 

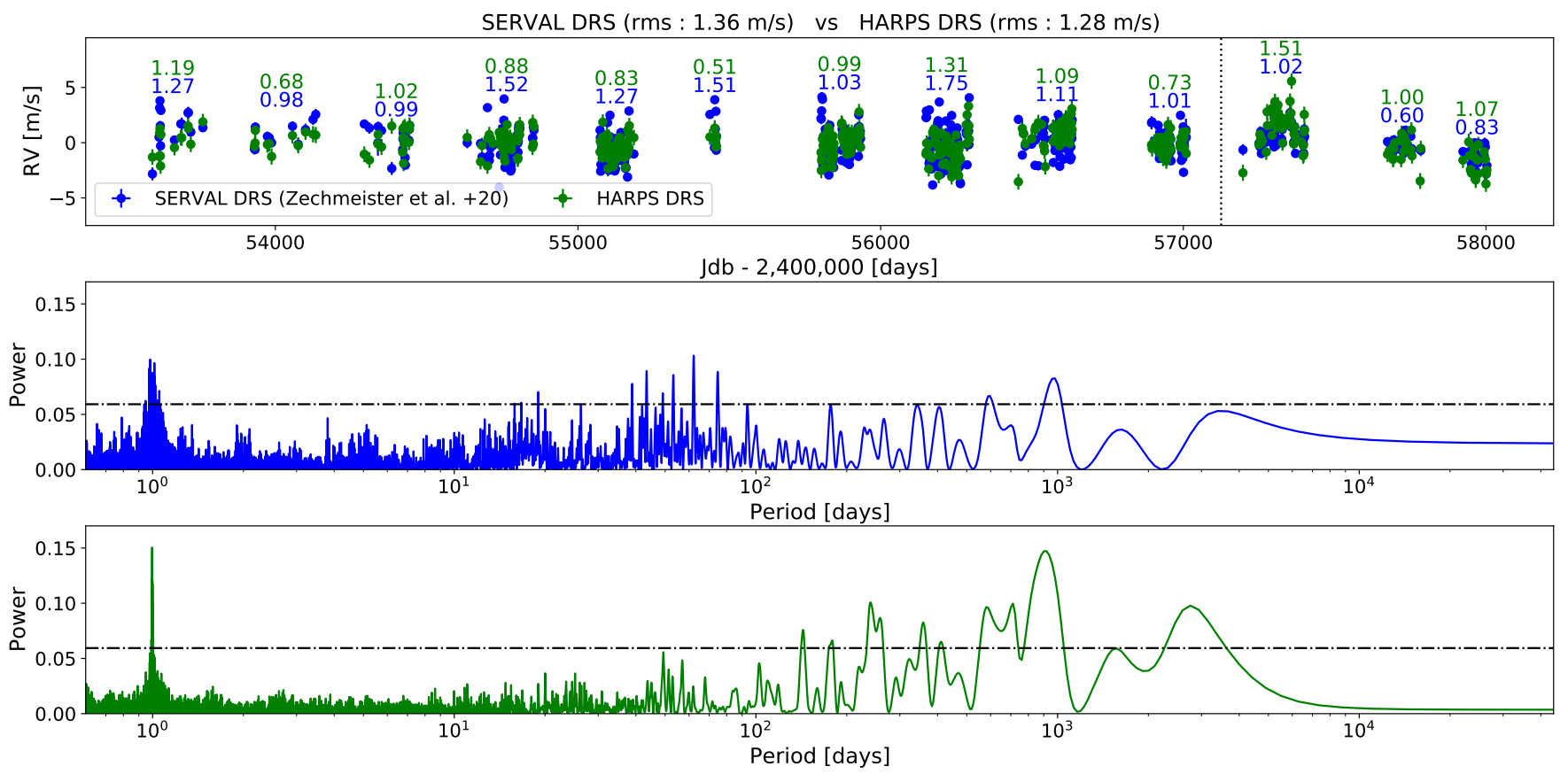

Fig. 10. Comparison between different reduction pipelines for 13 years of HD 10700 HARPS observations. First row: comparison between the SERVAL post-processing pipeline (without night-to-night offset correction; Trifonov et al. 2020; Zechmeister et al. 2020) time series (blue dots) and HARPS DRS (green dots). The weighted rms are indicated for each observational season as well as for the full time series in the title. The HARPS DRS time series present a significantly lower rms in each season before the fibre upgrade (vertical dotted line), whereas the opposite conclusion holds for SERVAL after the upgrade. Second row: GLS periodogram of the SERVAL time series. The $1 \%$ FAP level is indicated by the dotted-dashed line. Third row: same as the middle row but for HARPS DRS extracted time series.

\section{1. $H D 10700$}

We used HD 10700 as a star of reference to perform pipeline performance comparison and a planetary injection test. This star is known to be a particular inactive star maybe in a state similar to the Maunder minimum for the Sun (Lovis et al. 2011a). On 13 years of HARPS observations, the RV rms is observed to be $1.27 \mathrm{~m} \mathrm{~s}^{-1}$, which is an impressive proof of stellar and instrumental stability. Moreover, this system has been intensively followed by HARPS and contains no confirmed planet but only possible candidates with RV amplitudes lower than $1 \mathrm{~m} \mathrm{~s}^{-1}$, even if no agreement was found between different analyses (Pepe et al. 2011; Tuomi et al. 2013; Feng et al. 2017).

We first compared the publicly available RVs obtained with the SERVAL post-processing pipeline (Trifonov et al. 2020; Zechmeister et al. 2020) with the ones obtained by the HARPS DRS in Fig. 10. We note that in the publicly available SERVAL RVs, two different products are available. RVs computed using a stellar template matching technique, and the same RVs but corrected for night-to-night calibration offsets (e.g., Dumusque 2018) measured on the time series of several quiet stars. To have a fair comparison with the DRS RVs, we considered the SERVAL RVs without night-to-night offset correction. We see that for HD 10700, SERVAL does not performs as well as the DRS, with an RV rms over the entire time series of 1.36 compared to $1.27 \mathrm{~m} \mathrm{~s}^{-1}$, and the RV rms for each season being higher than for the DRS for the data before the change of the HARPS fibres in 2015 (Lo Curto et al. 2015). SERVAL is performing best after the fibre upgrade of the instrument. In addition, while SERVAL seems to slightly remove some signals with periods longer than a hundred days, it seems that more signals are present between 40 and 100 days, which is worrisome. It is clear from the Trifonov et al. (2020) paper that SERVAL performs better than the DRS for M-dwarfs since the stellar template-matching approach used by SERVAL is known to work better for these stars (Anglada-Escudé \& Butler 2012). However, the Trifonov et al. (2020) paper does not asses the performance of the code with respect to spectral type, and this example shows that for $G$ dwarfs, SERVAL does not necessarily outperform the DRS.

We then compared the SERVAL RVs with the ones obtained by YARARA. As YARARA works with nightly binned spectra, and thus RVs, we nightly binned the SERVAL data to perform the comparison. Two nights were clearly outliers in SERVAL reprocessed data, so we removed them in both datasets. In this case, to perform a fair comparison, we took the SERVAL RVs with the night-to-night offset correction applied. As seen in Fig. 11, correcting for the night-to-night offset significantly improves the SERVAL RVs in term of rms $\left(1.13 \mathrm{~m} \mathrm{~s}^{-1}\right)$; however, the forest of peaks between 40 and 100 days is still present. In comparison, the global RV rms is reduced down to $1.02 \mathrm{~m} \mathrm{~s}^{-1}$ after YARARA processing, which is equivalent to a quadratic difference improvement of $0.77 \mathrm{~m} \mathrm{~s}^{-1}$ compared to the HARPS DRS. We highlight in Fig. 12 for HD 10700, the different contaminations corrected by YARARA to show the relative strength of each component relative to the others. We can see that most of the contaminations are peak-to-peak larger than $10 \mathrm{~cm} \mathrm{~s}^{-1}$. The dominant systematics is produced by the stitching (Dumusque et al. 2015) with $1 \mathrm{~m} \mathrm{~s}^{-1}$ peak-to-peak variation.

By comparing Figs. 10 and 11, we can see that YARARA provides better individual season RV rms compared to the DRS for only half of the observational seasons, which highlights the difficulty to significantly improve the RV below $1 \mathrm{~m} \mathrm{~s}^{-1}$ on HARPS. Despite those differences, by looking at the global RV rms and the periodograms of the different time series, YARARA seems to clearly outperform SERVAL and the DRS. 
SERVAL (rms : $1.13 \mathrm{~m} / \mathrm{s})$ Vs YARARA (rms : $1.02 \mathrm{~m} / \mathrm{s})$
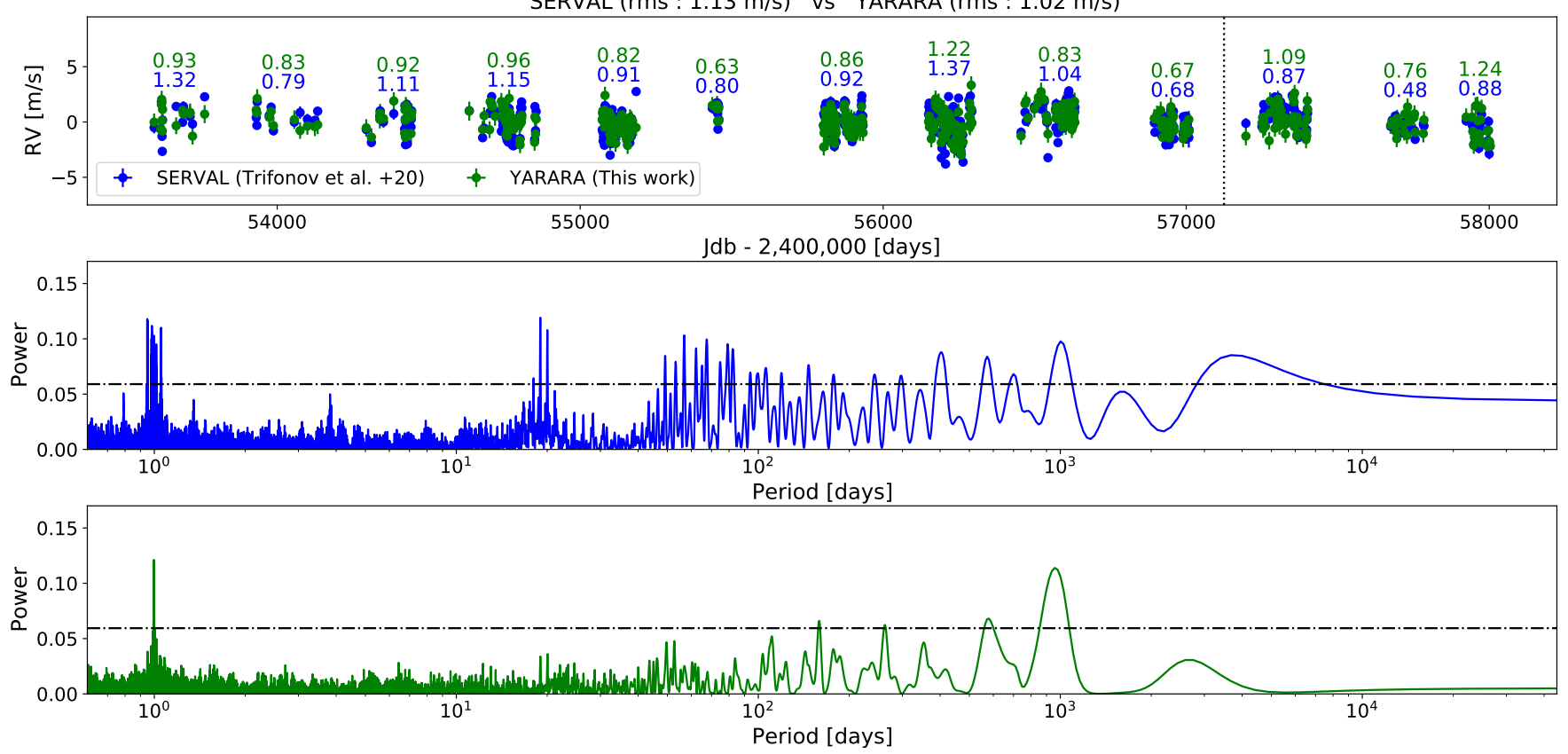

Fig. 11. Same comparison as Fig. 10, except that the comparison is now between YARARA time series (green dots) and the SERVAL postprocessing pipeline (with night-to-night offset correction, blue dots) presented in Trifonov et al. (2020). The rms is lower with YARARA for all the observational seasons before the fibre upgrade. The periodogram also presents a cleaner aspect.

YARARA seems to perform better in correcting for the different instrumental systematics, and thus give RVs with a smaller rms and that present a cleaner periodogram. However, this would be useless if YARARA processing absorbs planetary signals. To check that this was not the case, we injected into the spectra, before YARARA processing, the signature of two planets in circular orbits. A first one close the second harmonics of 1-year at 122.42 days and with an RV semi-amplitude of $K=3 \mathrm{~m} \mathrm{~s}^{-1}$, and a second one at 37.87 days with $K=2 \mathrm{~m} \mathrm{~s}^{-1}$. The first planet is equivalent to planet $\mathrm{f}$ in HD 10180, and since our analysis of HD 10180 (see Sect. 3.3) shows that the semi-amplitude of planet f change significantly after YARARA processing, we wanted to be sure that the recipes dealing with yearly instrumental effects were not perturbing the amplitude of planetary signals at the harmonics of a year.

To inject the planetary signals in the HD 10700 spectra, we proceeded as follows. First, we ran the HD 10700 data through YARARA, as described in Sect. 2, and we kept at each stage the contamination map. Secondly, merged 1D corrected spectra are shifted according to the planetary signals, before reintroducing the extracted contaminations. It would be wrong to simply shift the spectra still contaminated by systematics since the planetary signals would affect the instrument systematics. For example, telluric lines would not be fixed anymore in the BERV rest frame. Once the planets are properly injected in the original spectra, we ran again YARARA to check that the planetary signals are not absorbed.

Both injected planets were found to produce the highest peak in the generalised Lomb-Scargle (GLS) periodogram as expected from the high amplitudes of the planetary signals injected. However, to validate the efficiency of YARARA in recovering planetary signal while mitigating other systematics, we needed to measure at which precision the injected planetary signals were recovered. In Fig. 13, we compare the semiamplitude $K$ for the best-fitted Keplerian model for the two injected planets in the following RV time series: (1) DRS (blue marker) (2) before YARARA correction (yellow marker) (3) after YARARA flux correction (green marker) and (4) after YARARA morphological corrections and lines rejection (red marker). There are few differences between the RVs of the DRS and YARARA before correction, justifying their different treatment. YARARA is working with 1D-merged spectra while the DRS starts from 1D-echelle-order spectra. In addition, the colour correction is performed differently, the lines selected for the CCF mask are different, and the cross-correlation algorithm is different.

To determine an uncertainty on the semi-amplitude that could be due to pre-existing power in the time series, we fitted the same model on the DRS time series without the planet injected and reported the amplitudes of the signals as error bars. The relative uncertainties compared to the injected amplitudes is $7 \%$.

All the planetary semi-amplitudes recovered using the different datasets are compatible with the injected values. This is expected as the corrections performed by YARARA reduce the rms by $0.29 \mathrm{~m} \mathrm{~s}^{-1}$, whereas the amplitude of the injected signals are an order of magnitude larger, 2 and $3 \mathrm{~m} \mathrm{~s}^{-1}$. We notice that after YARARA correction, we are able to recover the exact amplitude of the injected 37.87-day signal. It is not the case for the 122.42 injected planet for which a value $~ 6 \%$ smaller is found. This smaller value, still compatible with the injected signal at one sigma, could be explained by the fact that the signal is at a harmonic of a year, and many different corrections in YARARA are mitigating systematics at a year. Thus, there could be small cross-talks between YARARA corrections and planetary signals close to a year or a harmonic of it. However, this test on HD 10700 shows that at most $10 \%$ in the semi-amplitude signals at one year or a harmonic of it could be absorbed by YARARA, which is in any case better than leaving perturbing yearly signals in the datasets. From this simulation of planet recovery, it seems that YARARA does not absorb significantly planetary signals in the final RV time series. Further tests on 

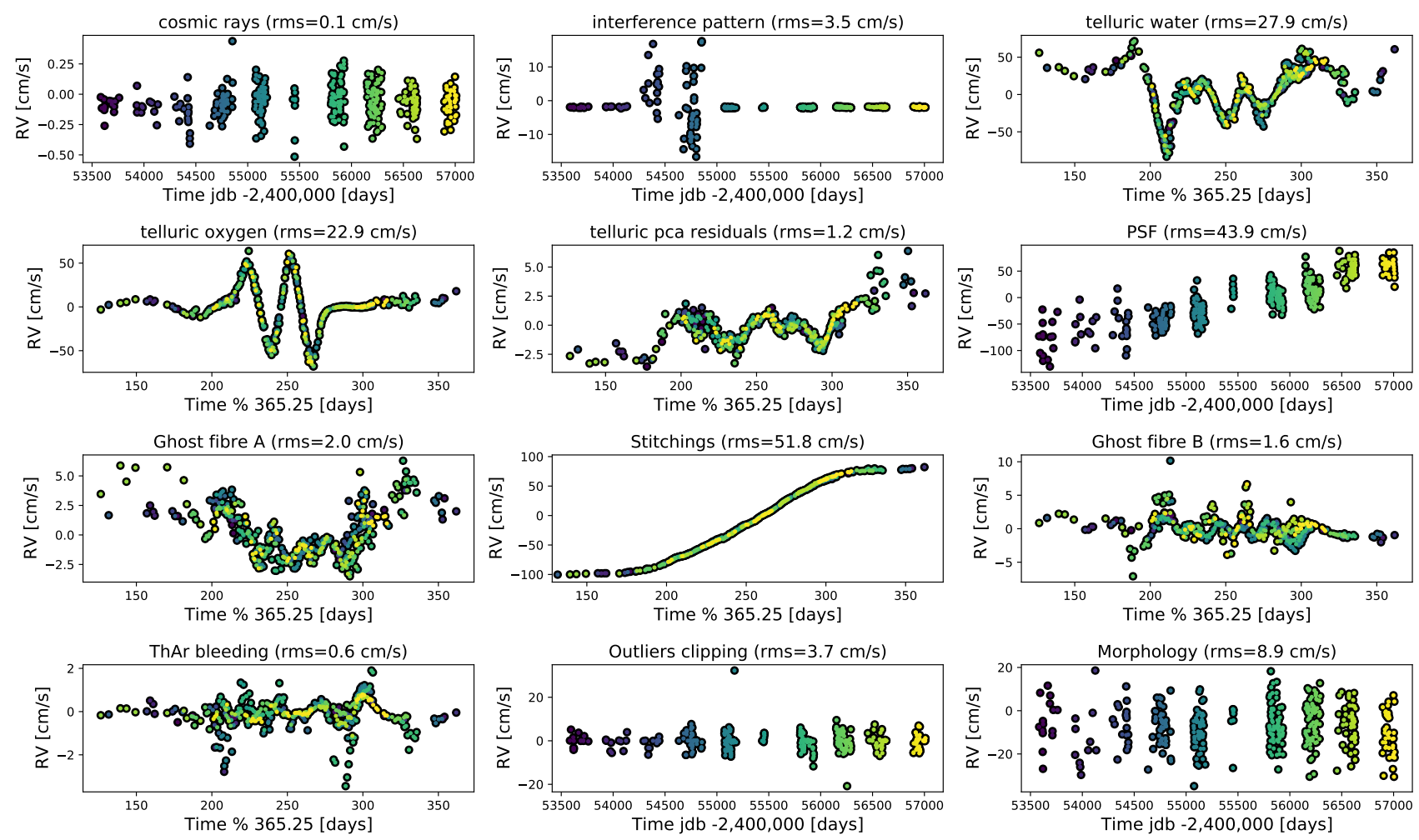

Fig. 12. Decomposition of the individuals components corrected in YARARA for HD 10700 on HARPS. Each RV component was obtained, as in Fig. 4, by computing the RVs before and after the flux corrections of a dedicated YARARA recipe. The RVs are obtained from the tailored line selection. Systematics known to be related to 1-year systematics are time-folded. We note that systematics can be folded at one sidereal year but are not described by sinusoid, explaining why their power can leak afterwards onto others frequencies in the periodogram. Despite the moderate $\mathrm{rms}$ of some components, the peak-to-peak of most of them is above $10 \mathrm{~cm} \mathrm{~s}^{-1}$.

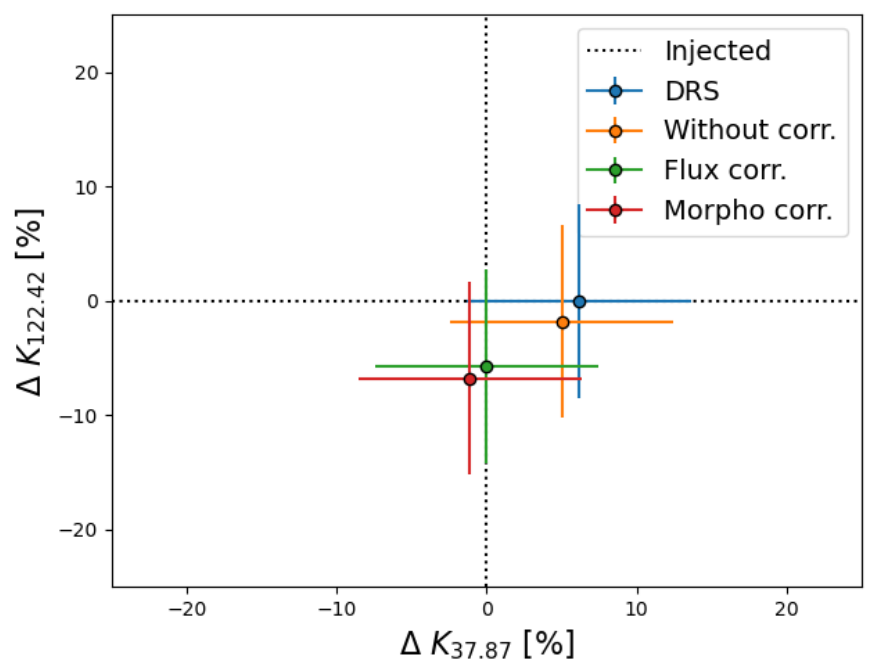

Fig. 13. Planetary injection recovery in the HD 10700 dataset; comparison between the injected $K$ semi-amplitudes (black dotted line) and the recovered ones obtained after fitting a two-sinusoid model for the planets at $37.87\left(K=2 \mathrm{~m} \mathrm{~s}^{-1}\right)$ and 122.42 days $\left(K=3 \mathrm{~m} \mathrm{~s}^{-1}\right)$. No significant absorption of the planetary signals is observed after YARARA processing, neither by flux correction (green markers) nor by morphological correction (red markers), since values similar to those without correction (orange markers) or DRS (blue markers) are found. All the recovered amplitudes are compatible with the injected values considering the uncertainty due to pre-existing signals.

other stellar systems, presented in the next two subsections, will confirm this conclusion.

\section{2. $H D 215152$}

HD 215152 is a compact system of four planets in close-in orbits ( $<30$ days) and close to a resonant configuration (Delisle et al. 2018). In that paper, the authors already reported a 1-year signal present in the RV data that was interpreted as mostly induced by the stitching of the HARPS detector. In order to remove this signal, the authors removed stellar lines crossing each stitching from the CCF mask. Therefore, their strategy consisted in masking instead of correcting for the signal. In this section, we demonstrate that by applying YARARA, the perturbing oneyear signal is completely mitigated without masking any spectral region, which has the advantage of improving the RV precision of the final dataset.

An interesting feature of YARARA resides in the fact that since the pipeline is applied sequentially, it is possible to quantify the RV perturbation of each contamination. To do so, the difference of the RV time series before and after the flux correction of each recipe is computed, directly providing the RV counterpart of the contamination. This method was used in Sect. 2.5 to highlight the instrument ageing on HD 10700. With YARARA, we confirm that stitchings are the main source of the one-year signal in the RV time series of HD 215152, with a peak-to-peak amplitude of $1 \mathrm{~m} \mathrm{~s}^{-1}$ as for HD 10700 (see Fig. 12).

In Fig. 14, we displayed the RV time series before and after YARARA corrections. The RV rms is decreased from 2.14 down to $1.57 \mathrm{~m} \mathrm{~s}^{-1}$ (equivalent to $1.45 \mathrm{~m} \mathrm{~s}^{-1}$ in quadratic difference). The GLS periodogram of each time-serie is showing that the 1 -year signal and its first harmonic are considerably reduced whereas the power at planetary periods (5.7, 7.3 and 10.5 days) are boosted. By looking at the RV difference, a clear linear trend, 

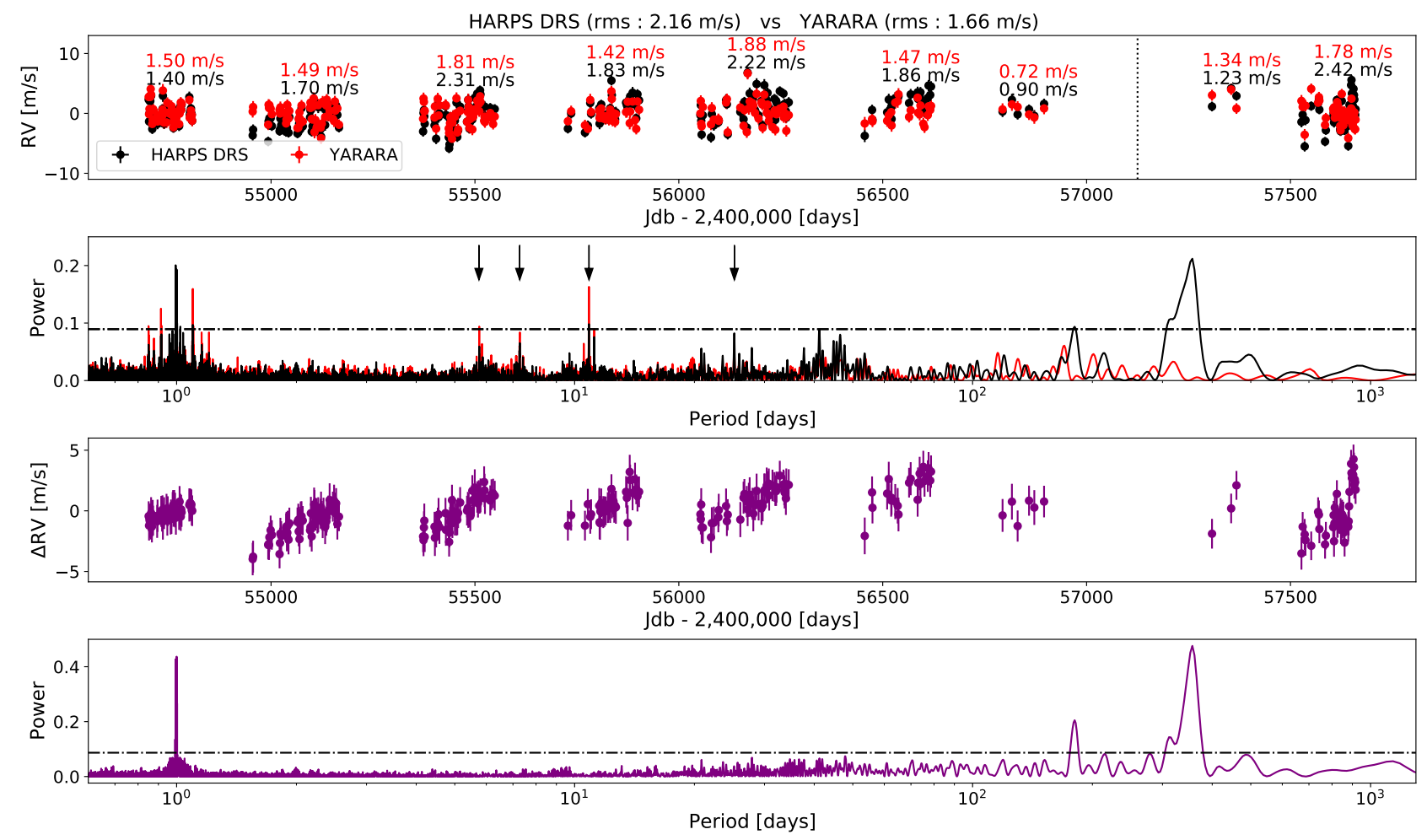

Fig. 14. Application of the YARARA post-processing on the HD 215152 HARPS dataset. First row: comparison of the RV time series extracted with the HARPS DRS (black dots) and after YARARA processing (red dots). Second row: GLS periodogram of the previous time series. The 1\% FAP level is indicated as a dotted-dashed line. Exoplanets reported in Delisle(2018) are indicated by black arrows. After YARARA processing, the power at the planetary periods $(5.8,7.3,10.9$ days) are boosted. Third row: RV difference between input and output data. fourth row: GLS periodogram of the RV difference. The removed contaminations were clearly producing most of the signals at one year and its first harmonic.

induced by the stitching effect (Dumusque et al. 2015), can be observed for each season. The jitter on smaller timescales is produced by the tellurics.

\section{3. $H D 10180$}

HD 10180 is a system composed of at least 6 exoplanets (Lovis et al. 2011b), with RV amplitudes larger than $1 \mathrm{~m} \mathrm{~s}^{-1}$. One of them, HD $10180 \mathrm{f}$, has a period of 122.7 days, thus very close from a harmonic of a year. We could therefore think that the $3 \mathrm{~m} \mathrm{~s}^{-1}$ amplitude of this planet is affected by the yearly HARPS detector stitching as this perturbing effect was only detected a few years later (Dumusque et al. 2015). Indeed, due to a complex interplay between real signals and data sampling, a yearly signal, such as the one induced by detector stitching, can present more power at a harmonic of a year. We therefore applied YARARA on HD 10180 HARPS observations to test if contaminating signals with one-year period can affect recovered planetary signals.

We note that a planetary candidate, HD $10180 \mathrm{~b}$, was also announced in Lovis et al. (2011b), but the induced signal with a period of 1.17 days and semi-amplitude of $80 \mathrm{~cm} \mathrm{~s}^{-1}$ is not visible in our analysis, probably due to night-binning. Since the same night-binning was performed for all the datasets that we compare in this section, the existence or not of this ultra-shortperiod planet is not relevant here.

After YARARA processing, the six exoplanets are well recovered, as we can see from the 11-periodogram ${ }^{6}$ (Hara et al.

\footnotetext{
6 The python code is freely accessible on GitHub : https ://gi thub com/nathanchara/l1periodogram
}

2017) in Fig. 15. This is another example that shows that YARARA is not absorbing planetary signals. As done for the planetary signal recovery test performed on HD 10700 (see Sect. 3.1), we compared in Fig. 16 the orbital parameters of the six planets obtained with the data from the DRS and with the data at a different step of YARARA's processing. Those parameters, with their respective error bars were obtained using a Markov chain Monte Carlo (MCMC) sampler. In contrast to HD 10700, no line was rejected by the 1-year criterion (Sect. 2.11), which is expected since the LBL precision is lower for HD 10180 due to spectra with lower $\mathrm{S} / \mathrm{N}$ (median $S / N \sim$ 160).

When comparing the recovered planetary orbital parameters, we do not observe any significant difference for the three innermost exoplanets. However, for the 122-day planet, its amplitude decrease by $0.55 \mathrm{~m} \mathrm{~s}^{-1}$ after the full YARARA processing compared to the DRS. This $18 \%$ drop is not compatible with the uncertainties. As a comparison, the amplitude of the planet injected at the same period and amplitude on HD 10700 (see Sect. 3.1) was only reduced by $7 \%$ after morphological corrections. Therefore, the decrease observed here seems to be real and is likely explained by the interference of the 122-day planetary signal with one-year systematics. This explanation is also favoured since in the YARARA input dataset, the planetary signal has an amplitude of $3.4 \mathrm{~m} \mathrm{~s}^{-1}$, which is $15 \%$ higher than the published value. This can be explained by the fact that in YARARA input dataset, all the regions of the stellar spectra are taken into account to measure the RV, even the parts strongly affected by tellurics, which is not the case for the DRS as the mask used for cross-correlation exclude regions of strong telluric contamination. This example of planet HD $10180 \mathrm{f}$ 
M. Cretignier et al.: YARARA: Significant improvement in RV precision through post-processing of spectral time series

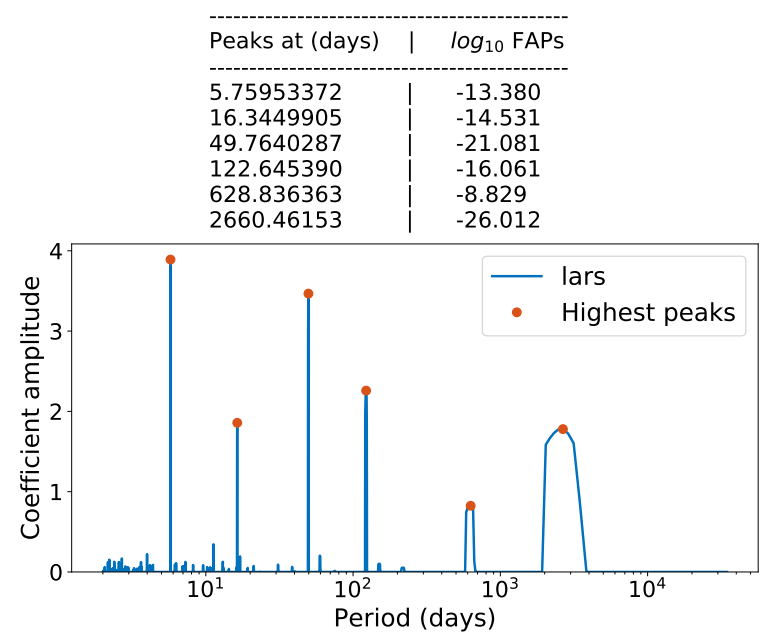

Fig. 15. L1 periodogram (Hara et al. 2017) applied to the YARARA post-processed time series of HD 10180. The six exoplanets in this stellar system (Lovis et al. 2011b) are recovered at high significance, as highlighted by the high FAP values.

demonstrates how some yearly contaminations can interfere with a planetary signal and bias the recovered orbital parameters. The minimum mass of HD $10180 \mathrm{f}$ should therefore be updated from $23.6 \pm 1.3 M_{\oplus}$ down to $19.4 \pm 1.2 M_{\oplus}$.

We can see other significant differences for HD $10180 \mathrm{~g}$ and $h$. Using DRS data, the 600-days planet presents a moderate eccentric orbit ( $e=0.37 \pm 0.13$ ), which motivated Lovis et al. (2011b) to fix the eccentricity of the planet to 0 in their model. After YARARA processing, the period shifts to 615 days and the eccentricity drops to $e=0.15 \pm 0.10$. Even if we cannot assess with certainty which configuration is the most favoured without performing dynamical stability simulations, it seems reasonable that the solution with the smallest eccentricity is favoured in this highly populated system. Regarding HD $10180 h$, the period increases to $P=2500$ days after YARARA processing, and the mass decreases from $52.2 \pm 3.3$ to $46.3 \pm 3.4 M_{\oplus}\left(65.3 \pm 4.6 M_{\oplus}\right.$ was reported in Lovis et al. 2011b).

We do not present here fully updated orbital parameters for HD 10180 as this will be the goal of a forthcoming paper. The idea of this section was to give an example that demonstrate that YARARA can help in characterising better planetary systems by cleaning non-planetary systematics in RV time series.

\section{Conclusion}

In this paper we have presented YARARA, a new postprocessing pipeline for deriving precise RV measurements from high-resolution spectra. The purpose of this pipeline is to provide better RV precision by correcting the spectral time series from different kinds of non-planetary systematics using a datadriven approach. The workflow of the code can be described as follows. All spectra in a time series are first normalised by RASSINE (Cretignier et al. 2020b). Then a master spectrum, as free as possible from systematics, is built, and all spectra are compared to this master. Regions that show strong flux residuals are cleaned either by modelling the signal using a PCA algorithm and then fitting for the first principal components, or by using a physically motivated model. From the obtained cleaned spectra, it is possible to derive RVs that are less contaminated by different instrumental and astrophysical systematics. We also demonstrate that analysing and correcting for the morphological variation in individual spectral lines after correcting for flux anomalies can further improve the RV precision.

YARARA has been designed to remove cosmic rays (Sect. 2.2), interference patterns (Sect. 2.3), tellurics (Sect. 2.4), PSF variation (Sect. 2.5), ghosts (Sect. 2.6), detector stitching effects (Sect. 2.7), reference fibre contamination (Sect. 2.8), and continuum precision (Sect. 2.9). To our knowledge, it is the first post-processing tool for high-resolution spectra that corrects for all these systematics at once.

By analysing the HARPS spectral time series of HD 10700 with YARARA, we demonstrate an instrumental stability over the entire lifetime of $1.02 \mathrm{~m} \mathrm{~s}^{-1}$ on nightly binned spectra (Sect. 3.1). Due to the extreme stability of HD 10700, we performed a planetary injection test on the spectral time series and found that planetary signals cannot be absorbed by more than $10 \%$ in semi-amplitude after YARARA processing. Then, by analysing HD 215152, we showed that the strong yearly signal induced by detector stitching is completely removed after YARARA processing, while the known planets in this system are not affected (Sect. 3.2). Finally, looking at HD 10180, all six detected planets are recovered with high significance (Sect. 3.3). Besides a significant change in semi-amplitude for planet $\mathrm{f}$, which is likely due to this planet having a period of 122 days and therefore being affected by the yearly effect of detector stitchings, all the other orbital parameters are compatible between the DRS and the final step of YARARA processing. A small change in the configuration of the outer part of the system is observed.

Since YARARA corrects for telluric contamination, excluding strong contaminated regions from cross-correlation masks to obtain RVs that are free from telluric contamination is no longer needed. Deriving RVs after YARARA processing can thus increase the $\mathrm{S} / \mathrm{N}$ and provide a better RV precision. The gain for the visible bandpass is rather small $(<10 \%$ Artigau et al. 2014), but could be huge in the near infrared (NIR). In addition, the correction of all the known systematics at the spectral level by YARARA is essential for performing further LBL analysis as contaminations can induce variation of hundreds of $\mathrm{m} / \mathrm{s}$ on individual spectral lines (see for instance the telluriccontaminated line in Fig. 3 of Cretignier et al. 2020a). Line-byline analysis is a promising path towards further understanding and mitigating stellar activity perturbations (e.g., Davis et al. 2017; Thompson et al. 2017; Wise et al. 2018; Dumusque 2018), and thus YARARA can be of great help in that respect.

Further improvement can be made on the pipeline. One contamination that has not yet been investigated is that induced by the moon reflection (e.g., Roy et al. 2020), which could theoretically be corrected for by training a PCA at the position of strong solar lines in the lunar RV rest frame. However, such contamination is expected to be very small for the high $\mathrm{S} / \mathrm{N}$ spectra of bright stars, which are the main input products for which YARARA will be efficient. Covariance between wavelength columns in spectral time series could also be accounted for, in particular to reduce the problems caused by some outliers during the multi-linear regressions.

In this paper we did not present any updated orbital parameters of the systems that we analysed, and we only mentioned the gain that can be achieved in the mass accuracy in certain cases, such as for HD $10180 \mathrm{f}$ with a mass $18 \%$ lower than what was found in Lovis et al. (2011b). An in-depth analysis of HARPS and HARPS-N observations using YARARA will be presented in the future.

Finally, YARARA is flexible and can be applied to the data of different highly stable spectrographs. The code is already implemented to work with HARPS, HARPS-N, CORALIE, 

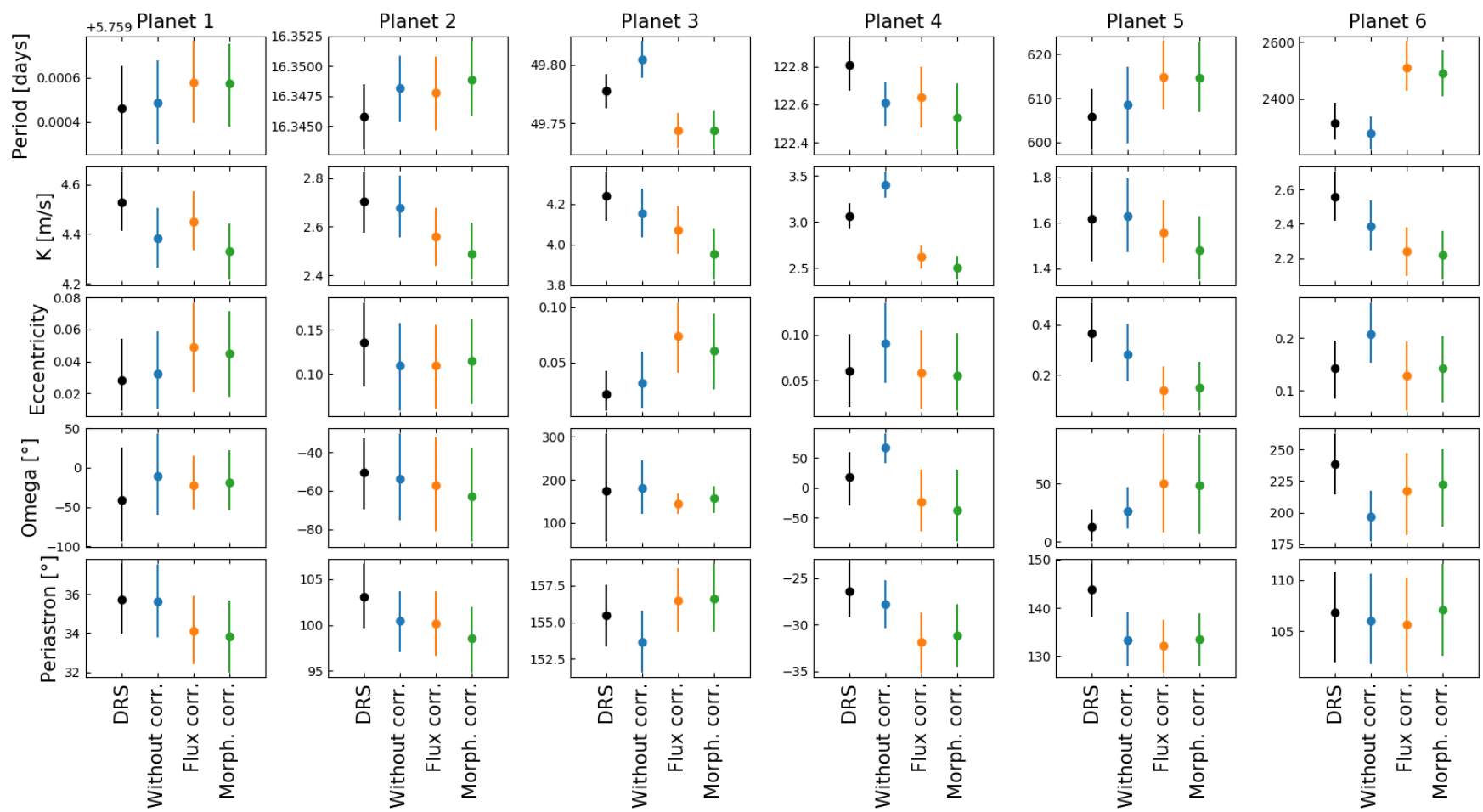

Fig. 16. Marginal distributions of the orbital parameters for the six exoplanets in the HD 10180 dataset obtained with an MCMC sampler. The different datasets analysed are encoded by different colours: the DRS (black dots), our reduction before flux correction (blue dots), after flux correction (orange dots), and after morphological correction (green dots). Each row represents one of the orbital parameters, and each column represents a planet.

CARMENES, EXPRES, and ESPRESSO spectra. Since the pipeline needs to construct a contamination-free master spectrum to highlight the contaminations before performing PCA, YARARA is expected to be more efficient for spectrographs working in the visible and for stars with large BERV coverage values. However, the pipeline could a priori also be employed for NIR spectra if combined with a model-driven approach to correct for tellurics.

Such methods of spectral time-series analysis can be employed for goals larger than the primary goal of RV precision; they could, for instance, be used in the context of stellar oscillations in order to detect and measure acoustic modes. In YARARA, spectra are usually binned nightly in order to boost the $\mathrm{S} / \mathrm{N}$, which is the main limitation of the pipeline. Therefore, it is unclear if such a tool could be adapted or not to detect the stellar pulsations of main sequence stars. It could, however, possibly be used in the context of longer and larger amplitude pulsation modes of variable stars, such as those of Cepheids (i.e., Anderson 2016).

Acknowledgements. We thank the referee Megan Bedell for her really helpful and constructive feedbacks. M.C. acknowledges the financial support of the SNSF. X.D is grateful to the Branco-Weiss Fellowship for continuous support. This project has received funding from the European Research Council (ERC) under the European Union's Horizon 2020 research and innovation program (grant agreement SCORE No. 851555). F. P. greatly acknowledges the support provided by the Swiss National Science Foundation through grant Nr. 184618. This publication makes use of the Data and Analysis Center for Exoplanets (DACE), which is a facility based at the University of Geneva $(\mathrm{CH})$ dedicated to extrasolar planets data visualisation, exchange and analysis. DACE is a platform of the Swiss National Centre of Competence in Research (NCCR) PlanetS, federating the Swiss expertise in Exoplanet research. The DACE platform is available at https://dace.unige.ch. This work has made use of the VALD database, operated at Uppsala University, the Institute of Astronomy RAS in Moscow, and the University of Vienna. This work has been carried out within the frame of the
National Centre for Competence in Research "PlanetS" supported by the Swiss National Science Foundation (SNSF).

\section{References}

Allart, R., Pino, L., Lovis, C., et al. 2020, A\&A, 644, A155

Anderson, R. I. 2016, MNRAS, 463, 1707

Anderson, R. I., Reiners, A., \& Solanki, S. K. 2010, A\&A, 522, A81

Anglada-Escudé, G., \& Butler, R. P. 2012, ApJS, 200, 15

Artigau, É, Astudillo-Defru, N., Delfosse, X., et al. 2014, Proc. SPIE, 9149, 914905

Bailey, S. 2012, PASP, 124, 1015

Baker, A., Blake, C., \& Sliski, D. 2017, Am. Astron. Soc. Meeting Abstr., 229, 155.15

Baliunas, S. L., Bennett, W., Briggs, J., et al. 1988, BAAS, 20, 994

Baranne, A., Queloz, D., Mayor, M., et al. 1996, A\&AS, 119, 373

Basri, G., Wilcots, E., \& Stout, N. 1989, PASP, 101, 528

Bedell, M., Hogg, D. W., Foreman-Mackey, D., Montet, B. T., \& Luger, R. 2019, AJ, 158, 164

Berdiñas, Z. M., Amado, P. J., Anglada-Escudé, G., Rodríguez-López, C., \& Barnes, J. 2016, MNRAS, 459, 3551

Berdyugina, S. V., \& Usoskin, I. G. 2003, A\&A, 405, 1121

Bertaux, J. L., Lallement, R., Ferron, S., \& Boonne, C. 2014, in 13th International HITRAN Conference, 8

Blake, C. H., \& Shaw, M. M. 2011, PASP, 123, 1302

Boisse, I., Bouchy, F., Hébrard, G., et al. 2011, A\&A, 528, A4

Bouchy, F., \& Carrier, F. 2001, A\&A, 374, L5

Bouchy, F., Pepe, F., \& Queloz, D. 2001, A\&A, 374, 733

Brandt, P. N., \& Solanki, S. K. 1990, A\&A, 231, 221

Cavallini, F., Ceppatelli, G., \& Righini, A. 1985, A\&A, 143, 116

Cegla, H. M., Watson, C. A., Shelyag, S., Mathioudakis, M., \& Moutari, S. 2019, ApJ, 879, 55

Coffinet, A., Lovis, C., Dumusque, X., \& Pepe, F. 2019, A\&A, 629, A27

Collier Cameron, A., Ford, E. B., Shahaf, S., et al. 2021, MNRAS, 505, 1699

Cretignier, M., Dumusque, X., Allart, R., Pepe, F., \& Lovis, C. 2020a, A\&A, 633, A76

Cretignier, M., Francfort, J., Dumusque, X., Allart, R., \& Pepe, F. 2020b, A\&A, 640, A42

Cunha, D., Santos, N. C., Figueira, P., et al. 2014, A\&A, 568, A35 
M. Cretignier et al.: YARARA: Significant improvement in RV precision through post-processing of spectral time series

Davis, A. B., Cisewski, J., Dumusque, X., Fischer, D. A., \& Ford, E. B. 2017, ApJ, 846, 59

de Laverny, P., Recio-Blanco, A., Worley, C. C., \& Plez, B. 2012, A\&A, 544, A126

Delisle, J. B., Ségransan, D., Dumusque, X., et al. 2018, A\&A, 614, A133

Dumusque, X. 2018, A\&A, 620, A47

Dumusque, X., Udry, S., Lovis, C., Santos, N. C., \& Monteiro, M. J. P. F. G. 2011, A\&A, 525, A140

Dumusque, X., Glenday, A., Phillips, D. F., et al. 2015, ApJ, 814, L21

Dumusque, X., Cretignier, M., Sosnowska, D., et al. 2021, A\&A, 648, A103

Errmann, R., Cook, N., Anglada-Escudé, G., et al. 2020, PASP, 132, 064504

Feng, F., Tuomi, M., Jones, H. R. A., et al. 2017, AJ, 154, 135

Fischer, D., Jurgenson, C., McCracken, T., et al. 2017, Am. Astron. Soc. Meeting Abstr., 229, 126.04

Gao, P., Plavchan, P., Gagné, J., et al. 2016, PASP, 128, 104501

Gray, D. F. 2005, The Observation and Analysis of Stellar Photospheres (Cambridge, UK: Cambridge University Press)

Grunblatt, S. K., Howard, A. W., \& Haywood, R. D. 2015, ApJ, 808, 127

Hara, N. C., Boué, G., Laskar, J., \& Correia, A. C. M. 2017, MNRAS, 464, 1220

Haywood, R. D., Collier Cameron, A., Queloz, D., et al. 2014, MNRAS, 443, 2517

Jones, D. E., Stenning, D. C., Ford, E. B., et al. 2017, ArXiv e-prints [arXiv:1711.01318]

Kerber, F., Rose, T., Chacón, A., et al. 2012, in Ground-based and Airborne Instrumentation for Astronomy IV, eds. I. S. Mc Lean, S. K. Ramsay, H. Takami, et al., SPIE Conf. Ser., 8446, 84463N

Kosiarek, M. R., \& Crossfield, I. J. M. 2020, AJ, 159, 271

Kurucz, R. L. 2005, Mem. Soc. Astron. It. Suppl., 8, 14

Lafarga, M., Ribas, I., Lovis, C., et al. 2020, A\&A, 636, A36

Leet, C., Fischer, D. A., \& Valenti, J. A. 2019, AJ, 157, 187

Li, D., Blake, C. H., Nidever, D., \& Halverson, S. P. 2018, PASP, 130, 014501

Lo Curto, G., Pepe, F., Avila, G., et al. 2015, Messenger, 162, 9

Löhner-Böttcher, J., Schmidt, W., Schlichenmaier, R., Steinmetz, T., \& Holzwarth, R. 2019, A\&A, 624, A57

Lovis, C., Dumusque, X., Santos, N. C., et al. 2011a, ArXiv e-prints [arXiv:1107.5325]

Lovis, C., Ségransan, D., Mayor, M., et al. 2011b, A\&A, 528, A112

Malavolta, L., Lovis, C., Pepe, F., Sneden, C., \& Udry, S. 2017, MNRAS, 469 3965

Mayor, M., Pepe, F., Queloz, D., et al. 2003, Messenger, 114, 20

Ning, B., Wise, A., Cisewski-Kehe, J., Dodson-Robinson, S., \& Fischer, D. 2019, AJ, 158, 210

Nordlund, A., Stein, R. F., \& Asplund, M. 2009, Liv. Rev. Sol. Phys., 6, 2

Oranje, B. J. 1983, A\&A, 124, 43
Palacios, A., Gebran, M., Josselin, E., et al. 2010, A\&A, 516, A13 Peck, A. 2020, Mirror, 1, 37

Pepe, F., Mayor, M., Rupprecht, G., et al. 2002a, Messenger, 110, 9 Pepe, F., Mayor, M., Galland, F., et al. 2002b, A\&A, 388, 632

Pepe, F., Bouchy, F., Queloz, D., \& Mayor, M. 2003, in Scientific Frontiers in Research on Extrasolar Planets, eds. D. Deming, \& S. Seager, ASP Conf. Ser., 294, 39

Pepe, F., Lovis, C., Ségransan, D., et al. 2011, A\&A, 534, A58

Pepe, F., Molaro, P., Cristiani, S., et al. 2014, Astron. Nachr., 335, 8

Pepe, F., Cristiani, S., Rebolo, R., et al. 2021, A\&A, 645, A96

Povich, M. S., Giampapa, M. S., Valenti, J. A., et al. 2001, AJ, 121, 1136

Queloz, D., Henry, G. W., Sivan, J. P., et al. 2001, A\&A, 379, 279

Rajpaul, V. M., Aigrain, S., \& Buchhave, L. A. 2020, MNRAS, 492, 3960

Reiners, A., Shulyak, D., Anglada-Escudé, G., et al. 2013, A\&A, 552, A103

Rieutord, M., Roudier, T., Rincon, F., et al. 2010, A\&A, 512, A4

Roy, A., Halverson, S., Mahadevan, S., et al. 2020, AJ, 159, 161

Sanchez, J. A., Seifahrt, A., Bean, J., \& Kasper, D. 2021, Am. Astron. Soc. Meeting Abstr., 53, 239.08

Seifahrt, A., Käufl, H. U., Zängl, G., et al. 2010, A\&A, 524, A11

Seifahrt, A., Stürmer, J., Bean, J. L., \& Schwab, C. 2018, in Ground-based and Airborne Instrumentation for Astronomy VII, eds. C. J. Evans, L. Simard, \& H. Takami, SPIE Conf. Ser., 10702, 107026D

Smette, A., Sana, H., Noll, S., et al. 2015, A\&A, 576, A77

Stenflo, J. O., \& Lindegren, L. 1977, A\&A, 59, 367

Thompson, A. P. G., Watson, C. A., de Mooij, E. J. W., \& Jess, D. B. 2017, MNRAS, 468, L16

Tody, D. 1986, in Instrumentation in Astronomy VI, ed. D. L. Crawford, Proc. SPIE, 627, 733

Tody, D. 1993, in Astronomical Data Analysis Software and Systems II, eds. R. J. Hanisch, R. J. V. Brissenden, \& J. Barnes, ASP Conf. Ser., 52, 173

Trifonov, T., Tal-Or, L., Zechmeister, M., et al. 2020, A\&A, 636, A74

Tuomi, M., Jones, H. R. A., Jenkins, J. S., et al. 2013, A\&A, 551, A79

Ulmer-Moll, S., Figueira, P., Neal, J. J., Santos, N. C., \& Bonnefoy, M. 2019, A\&A, 621, A79

Upton, G., \& Cook, I. 1996, Oxford University Press, 443

Vázquez Ramió, H., Régulo, C., \& Roca Cortés, T. 2005, A\&A, 443, L11

Wildi, F., Pepe, F., Chazelas, B., Lo Curto, G., \& Lovis, C. 2011, SPIE Conf Ser., 8151, 81511F

Wilson, O. C., \& Vainu Bappu, M. K. 1957, ApJ, 125, 661

Wise, A. W., Dodson-Robinson, S. E., Bevenour, K., \& Provini, A. 2018, AJ, 156,180

Xu, X., Cisewski-Kehe, J., Davis, A. B., Fischer, D. A., \& Brewer, J. M. 2019, AJ, 157, 243

Zechmeister, M., Reiners, A., Amado, P. J., et al. 2020, SERVAL: SpEctrum Radial Velocity AnaLyser 


\section{Appendix A: Telluric line location extraction}

The detailed correction of telluric lines described in Sect. 2.4 is performed by fitting a PCA in the terrestrial rest frame at the telluric lines' location. To do so, the main step required is therefore to select the so-called locations, or, said differently, to be able to form a sub-selection of wavelengths $\lambda_{j}$ on which to fit the PCA.

Theoretically, this step could be done by simply selecting all the wavelengths known to contain telluric lines deeper than a fixed threshold in percent based on specific database (e.g., MolecFit Smette et al. 2015). Such a strategy contains three main disadvantages. First, the pipeline would no longer be selfsufficient; this goes against the main philosophy of YARARA, which is to depend as little as possible on static products. This allows for a relatively simple adaptation of YARARA on any spectrograph, independently of their available spectral range. Secondly, since telluric lines change in depth, any selection based on a depth threshold from a fixed template is somewhat arbitrary. Lastly, the main issue of such a strategy is to be independent of the $\mathrm{S} / \mathrm{N}$ of the observations.

By nature, any data-driven method cannot outperform the noise level of the observations. When performing a PCA analysis, it is not sufficient to guaranty that a telluric line is present at a specific wavelength column $\lambda_{j}$, we also have to guaranty that the $\mathrm{S} / \mathrm{N}$ of the signal is sufficient, and this cannot be produced by a static product since it will depend on the $\mathrm{S} / \mathrm{N}$ of the observations. Again, such conditions should be computed directly from the observations.

To know if the $\mathrm{S} / \mathrm{N}$ of a telluric line is sufficient, we can proceed as follow. It should be noted that PCA are not sensitive to offsets but only to the variance around the offset. The variance can be interpreted as the first order variation, whereas the offset as the zeroth order variation. In order to assess if the $\mathrm{S} / \mathrm{N}$ of a telluric line was sufficient, we then only selected wavelengths for which the zeroth order variation was detectable.

Detecting the zeroth order variation is quite straightforward. To do so, the ratio time series uncorrected from the telluric CCFs moments described in Sect. 2.4 is shifted in the terrestrial rest frame and the time-median of the time series is taken to create a master telluric spectrum. A 1.5 interquartile sigma clipping was then performed to detect all the outliers that are proxies of the telluric locations. Such a method was also used to form the wavelength set $\lambda_{j}$ on which to train the PCA for the ThAr bleeding (see Sect. 2.8). The advantage of such a data-driven method is that only the telluric lines with a high enough $\mathrm{S} / \mathrm{N}$ will be selected. It improves the efficiency of the PCA, which is not trained on noisy data.

Using the data of HD 10700, we compared our telluric detection criterion with the MolecFit database in order to determine the completeness of our algorithm. To do so, we considered a MolecFit line as detected if this line was falling in a telluric region flagged by our pipeline. The cumulative distribution of the undetected telluric lines is displayed in Fig. A.1. As a comment, such an analysis is a standard product of the pipeline, therefore allowing us to easily check if something wrong occurred and the expected level of correction reached, usually defined as the $90 \%$ completeness level.

We see that all the lines deeper than $0.81 \%$ are detected and $90 \%$ of the undetected telluric lines are shallower than $0.08 \%$ coherent with the noise level of the observations $(0.21 \%$ in the red, see bottom panel Fig. 2). For HD 10180 and HD 215152, the two other stars presented in the paper, the $90 \%$ completeness level was situated respectively at $0.20 \%$ and $0.25 \%$, still below the percent level.

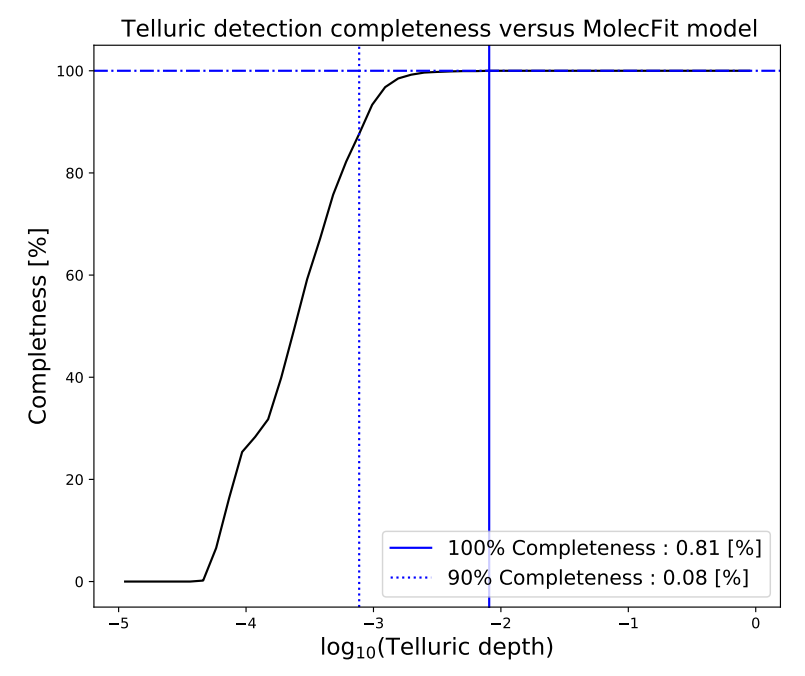

Fig. A.1. Computation of tellurics completeness of our detection algorithm compared to the MolecFit database for HD 10700. We show here the cumulative distribution of the MolecFit tellurics lines undetected by our algorithm. We see here that all tellurics lines deeper than $0.81 \%$ are detected. In addition, $90 \%$ of the undetected telluric lines all present depths shallower than $0.08 \%$, which is coherent with the average noise dispersion around $0.21 \%$ for HD 10700 (see the bottom panel of Fig. 2).

\section{Appendix B: Ghost modelling and location extraction}

In order to localise the ghosts on the detector, we first stacked two months of FLAT raw frames for fibres A and B, which allows the ghosts produced by the science and the simultaneous reference fibres to be identified. We then masked the main echelle orders using the order localisation provided by the DRS. In the master raw frames obtained (see left panel of Fig. 5), we can see several families of ghosts that are characterised by their angle with respect to the main echelle orders.

To localise the exact position of the ghosts, we first measured their relative angle with respect to the main echelle orders. This was done by incrementally rotating the raw master image while interpolating it on the initial pixel grid and summing up the intensities of each column. By plotting the summed intensity as a function of rotational angle, clear peaks appear at certain angles, corresponding to the different families of ghosts. We detected three families of ghosts, one with a relative positive angle, and two others with relative negative angles. We only considered at this stage the family of ghost that presented the highest flux (the one with the negative relative angle closest to $(0)$ as the other families were very dim and we could not see any impact of them in our river diagrams.

From the rotational angle and the position of the highest peaks, we can recover the position of the ghosts on the detector as straight lines. As ghosts do present a parabolic shape, we then looked for the maximum intensity around our linear approximation to obtain a localisation map for the ghosts. For each ghost, we extracted the flux at the localised position, and fitted a Gaussian profile. At the end of this process, we obtained the 2D map of ghost contamination seen in the middle panel of Fig. 5. Finally, we selected the region where ghosts contaminate the science fibre by computing the intersection between the main orders and the ghost model (last panel Fig. 5), and measured the relative intensity of ghosts by computing the ratio between the 
M. Cretignier et al.: YARARA: Significant improvement in RV precision through post-processing of spectral time series
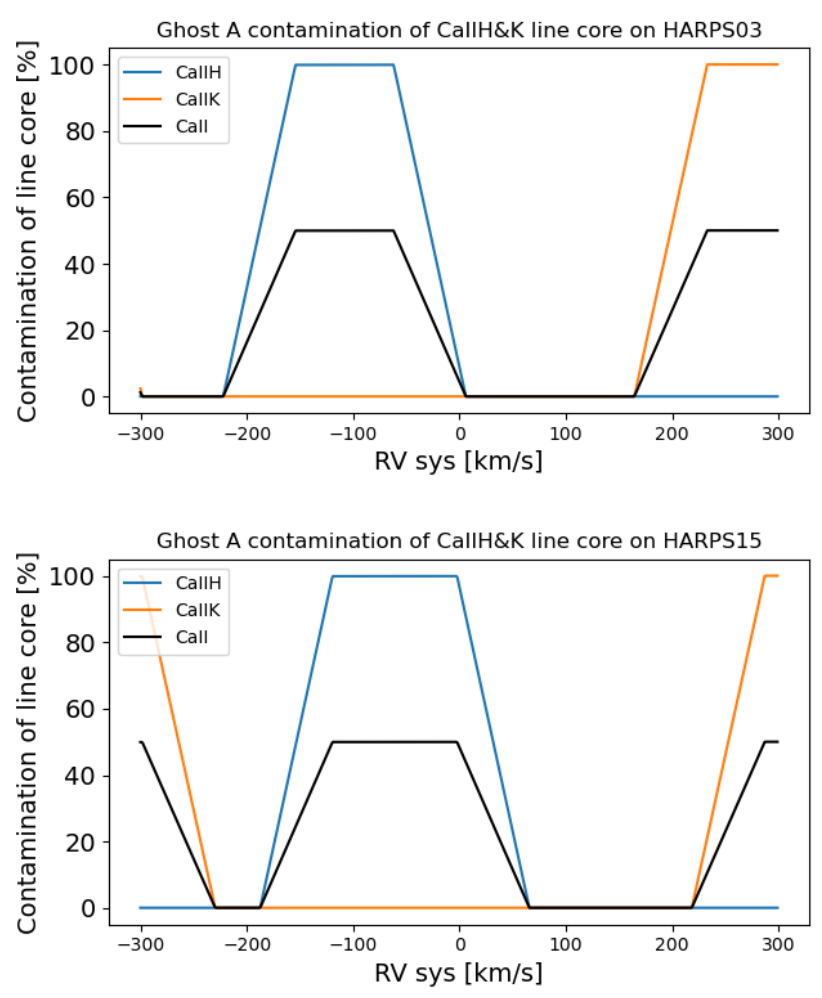

Fig. B.1. Computation of the covering fraction of ghosts from fibre A on the core of CaIIH\&K lines as a function of the systemic stellar velocity, $\mathrm{RV}_{\text {sys. }}$ Top: HARPS before fibre upgrade. Bottom: HARPS after fibre upgrade.

physical order and the ghost model intensities ${ }^{7}$. This process was applied on the FLAT master frames for fibres A and B in order to discriminate the ghosts originating from the science and the calibration fibres.

Such a static product can be used to measure the ghost contamination on the cores of the active CaII H\&K lines, where the contamination depends solely on the stellar systemic velocity $\mathrm{RV}_{\text {sys }}$ that will change the relative position between the lines' cores and the ghosts. On HARPS, only the ghosts from fibre A are sufficiently bright in that spectral region to be concerning. We extracted both chromospheric lines in short wavelength rectangular band-pass defined as $3933.66 \pm 0.45$ and $3968.47 \pm 0.45 \AA$ for the $K$ and $H$ lines, respectively. By shifting those filters with different $\mathrm{RV}_{\text {sys }}$ and computing the intersection with the ghost localisation, we can measure the covering fraction of ghosts on the cores of active lines as displayed in Fig. B.1. We see that during the full lifetime of HARPS, ghosts never contaminated simultaneously the core of both chromospheric lines. We note that such an analysis does not consider the relative flux variation in the ghosts and cores of the strong lines. Therefore, even a line completely covered by a ghost may present only a small relative contamination at the end if the CaII lines present a strong flux variation.

\section{Appendix C: Continuum absorption correction}

Our input products for YARARA are merged 1D spectra normalised by RASSINE, an alpha-shape algorithm valid under the assumption that the local maxima are probing the real continuum. As a consequence, an inaccurate continuum is often obtained for the blue part of the spectrum of $G-K$ dwarfs or everywhere for $M$-dwarfs since this condition does not hold. Given the stellar parameters of a given star, it is possible to correct for continuum absorption using a stellar template. We used here the freely available POLLUX database (Palacios et al. 2010) that contains normalised high-resolution spectra produced by MARCS (de Laverny et al. 2012) and ATLAS (Kurucz 2005) stellar atmosphere models. We note that MARCS models are 3D atmospheric models compared to 1D ATLAS models, but the ATLAS model grid of model is more extended and cover with a better resolution the $T-\log (g)$ space.

We show in Fig. C.1 an example of continuum absorption correction for GJ576, an M1.5 dwarf. The first step consists in getting an approximate value for the effective temperature and surface gravity of GJ576. This is done by minimising the $\chi^{2}$ between the model and three strong lines in our RASSINE master spectrum, namely $H_{\alpha}$, the $\mathrm{Na}$ doublet and the MgI triplet since the broad wings of those lines are known to be pressure and temperature sensitive (Gray 2005). We note that before minimising the $\chi^{2}$ between different models, the master spectrum is shifted in the stellar rest frame and the stellar templates are interpolated on the same wavelength grid as the master. We then performed a rolling 90 percentile with a window of $10 \AA$ on the stellar template and kept only the points above the obtained curve. Finally, only the closest maxima to the RASSINE anchors points are kept and a cubic interpolation is performed between these local maxima before applying a Savitchy-Golay filtering with a window of $10 \AA$. The obtained continuum (see orange curve in Fig. C.1) is used to divide the RASSINE master spectrum (black curve top panel), which allows the continuum absorption to be corrected for, as we can see in the bottom panel.

\footnotetext{
7 We note that this relative intensity is the one computed for the spectrum of a Tungsten lamp, which has its own spectral energy distribution. It is likely that for a stellar spectrum the relative intensity will be slightly different.
} 
Before correction

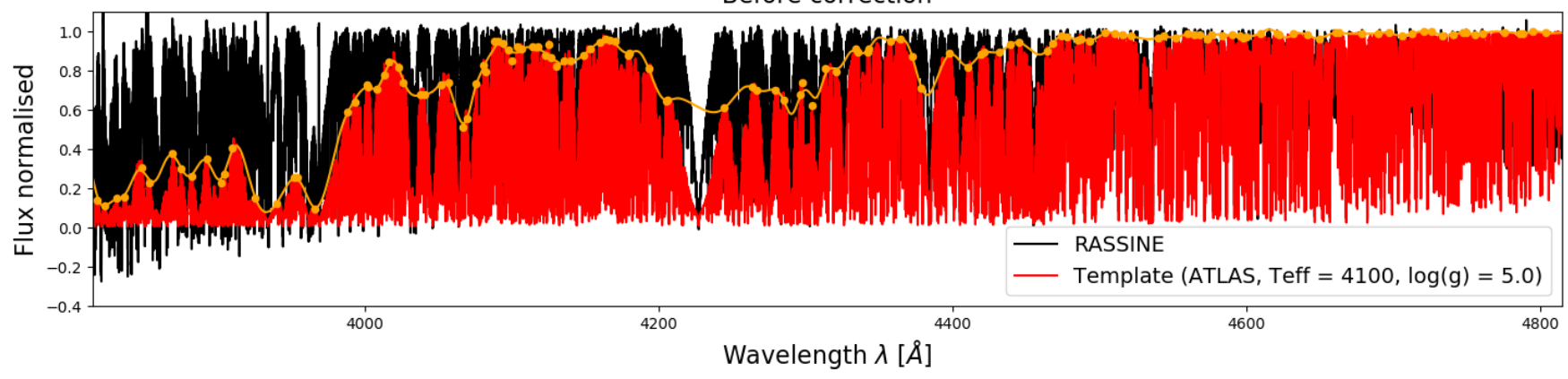

After correction

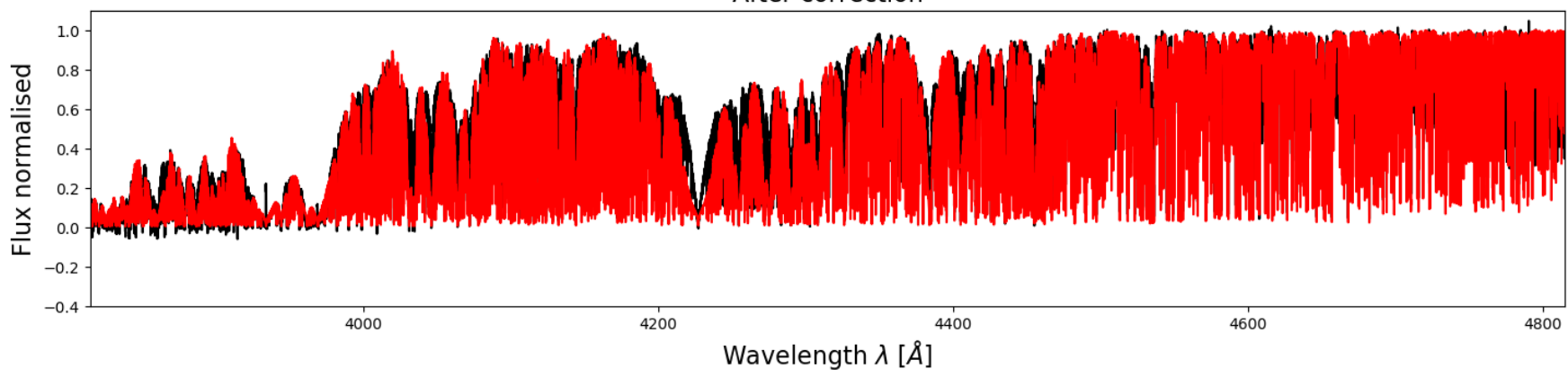

Fig. C.1. Correction of the absolute continuum level in the extreme blue of a cool star. Top: comparison between the GJ576 master spectrum coming out from RASSINE (black curve) and the best-fitted template model (red curve). The star is an M1.5 dwarf for which the best stellar template in ATLAS is a model with $T=4100 \mathrm{~K}$ and $\log (g)=5.0$. We show, in orange, the correction curve for the continuum absorption. Bottom: RASSINE master spectrum after template correction. The accuracy in the blue is considerably improved. 